Article

\title{
Ancient Ceramic Casting Molds from the Southern Russian Far East: Identification of Alloy Traces via Application of Nondestructive SEM-EDS and pXRF Methods
}

\author{
Irina S. Zhushchikhovskaya ${ }^{1, *}$ and Igor Yu. Buravlev ${ }^{2,3}$ (D) \\ 1 Institute of History, Archaeology \& Ethnology of Peoples of Far East, Far Eastern Branch of Academy of \\ Sciences, 690001 Vladivostok, Russia \\ 2 Institute of Chemistry, Far Eastern Branch of Academy of Sciences, 690022 Vladivostok, Russia; \\ buravlev.i@gmail.com \\ 3 Far Eastern Federal University, Russky Island, 690922 Vladivostok, Russia \\ * Correspondence: irina1zh@mail.ru
}

check for

updates

Citation: Zhushchikhovskaya, I.S.; Buravlev, I.Y. Ancient Ceramic Casting Molds from the Southern Russian Far East: Identification of Alloy Traces via Application of Nondestructive SEM-EDS and pXRF Methods. Heritage 2021, 4, 2643-2667. https://doi.org/10.3390/

heritage 4040149

Academic Editor: Chiara Soffritti

Received: 30 August 2021

Accepted: 22 September 2021

Published: 24 September 2021

Publisher's Note: MDPI stays neutral with regard to jurisdictional claims in published maps and institutional affiliations.

Copyright: (C) 2021 by the authors Licensee MDPI, Basel, Switzerland. This article is an open access article distributed under the terms and conditions of the Creative Commons Attribution (CC BY) license (https:/ / creativecommons.org/licenses/by/ $4.0 /)$.

\begin{abstract}
The investigation presented in this paper is a unique assemblage of ceramic casting molds discovered at one of the sites from the Bohai period (698-926) in the territory of the southern Russian Far East. The main research aim is to recognize probable traces of metal alloys cast in ceramic molds. Nondestructive pXRF and SEM-EDS methods were used as the research instruments for detecting the expected alloys' chemical components. As a result, the elements $\mathrm{Pb}, \mathrm{Sn}, \mathrm{Cu}$, and $\mathrm{As}$ were indicated at the surfaces of the molds' cavities with evidence of carbonization caused by the casting process. Preliminarily, two groups of alloys were distinguished: lead-bearing alloys and lead-free alloys. Our new insights are in good accordance with the results of previous investigations on chemical compositions of bronzes from the Bohai period archaeological sites of the southern Russian Far East. In particular, data on the examination of ceramic molds confirm the conclusion that various kinds of copper alloys were known and used in the bronze casting craft of the Bohai period.
\end{abstract}

Keywords: archaeological remains; Bohai State period (698-926); bronze casting; metalworking ceramics; SEM-EDS method; pXRF method; chemical composition; microstructure

\section{Introduction}

Fired-clay metal-casting molds are generally recognized as being one of the earliest kinds of technical ceramics. Their recovery from archaeological sites potentially provides important direct evidence for early metalworking practices. The examination of these artifacts focuses, at first, on their morphological and technological features and properties. The usage of physicochemical methods, in particular, binocular and petrography microscopy, SEM-EDS analysis, XPS analysis, X-ray tomography, FTIR analysis, and some others, provide objective information on ceramic fabrics and firing regimes of casting molds [1-8].

A relatively new research direction is the identification of preserved alloy traces at the surfaces of archaeological ceramic casting molds. An alloy's chemical composition traces may be detected by the application of natural science methods, in particular XRF analysis, in the examination of a ceramic mold's surface in contact with hot liquid metal. Physicochemical methods applied to casting ceramic mold examination allow us to detect the presence of basic alloy elements but not their original concentrations. Despite this, recognizing the alloy traces in ceramic molds is interesting and important archaeological evidence of the metalworking history. This refers to cases when discovered molds are single instances of the evidence of old metal processing and metalworking activities for studied archaeological sites or cultural communities $[7,9,10]$.

The research area considered in the article is the Primorye region, the main continental part of the southern Russian Far East bordering Northeast China and the Korean peninsula. 
At present, only two archaeological sites with casting molds are known in this area. A small series of stone-casting molds dated preliminary to around the border of $1 \mathrm{mil}$ BC-1 mil AD was unearthed at the multilayered settlement Siniye Skaly at the eastern seacoast of Primorye [11] (pp. 48-49). Another site is the multilayered settlement Kruglaya Dolina, also known as the Novogordeevka village settlement, in the central Primorye region (Figure 1). This archaeological site located in an area with modern agricultural activity was destroyed significantly. Poorly preserved remains of a metalworking workshop were discovered here in the early 1970s. The workshop was dated supposedly to the times when a large part of modern Primorye region's territory was made up of the Bohai Kingdom (698-926 AD) as the first state in Northeast Asia. The assemblage of bronze-casting inventory from the workshop's remains contain representative series of ceramic casting molds, series of ceramic crucibles, and one ceramic nozzle. Meanwhile, no finished bronze artifacts that might have been produced in the discovered molds were found at the site Kruglaya Dolina and at the neighboring walled settlement of Bohai period Kruglaya Sopka, known also as the Novogordeevka walled settlement [11] (pp. 71-72).

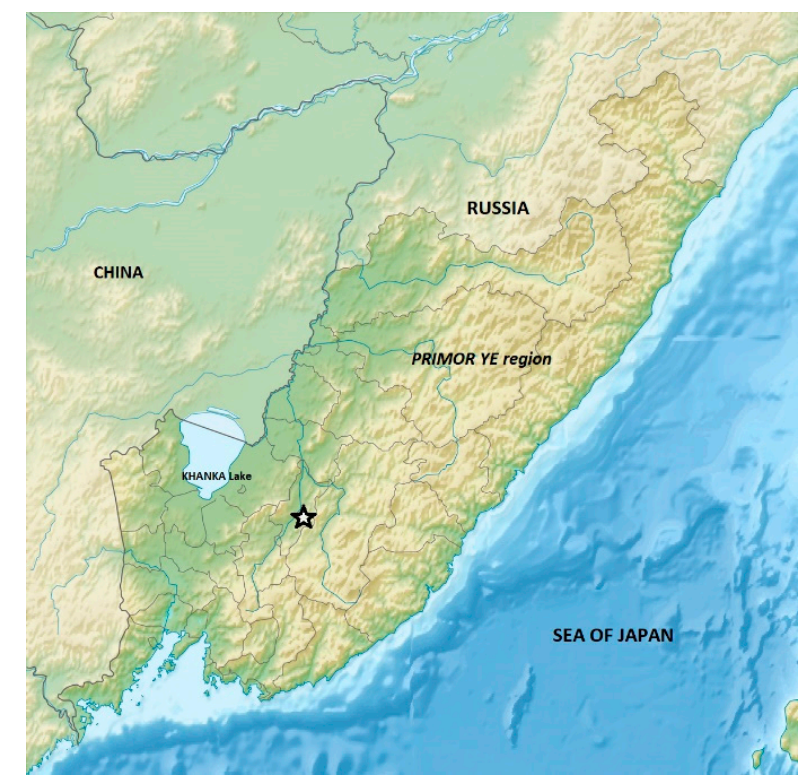

Figure 1. Map of the research area (Primorye region) with the location of the Kruglaya Dolina site (the "star" mark).

Before today, two categories of archaeological artifacts were studied as the sources of information on the Bohai State period bronze-casting technology and alloy compositions. At first, there were bronzes, mainly types of ornaments, unearthed at various Bohai sites of the southern Russian Far East. The investigation of Bohai bronzes, in particular alloys compositions, was conducted in the 1980s using the method of chemical semi-quantitative spectral analysis [11] (pp. 53-72). Some new examinations of Bohai period bronze chemical composition using pXRF and microscopy methods were provided in the last decade [12,13]. Another category of studied objects is ceramic crucibles from the Kruglaya Dolina site noted above. The investigations of metal residues from the crucibles were provided with the same chemical semi-quantitative spectral analysis method as was applied to the bronze items [11] (pp. 60-62). In general, for the Bohai period bronze-casting technology, several copper-alloy systems were determined based on the detection of the elements $\mathrm{Cu}, \mathrm{Pb}, \mathrm{Sn}$, and As and their quantitative ratios.

Ceramic casting molds from the Kruglaya Dolina site (the Novogordeevka village settlement) are being tested as a new potential source of information about copper alloys. Just recently, restricted series of molds were examined to identify the alloy traces at the ceramic surfaces. The primary research methods applied to casting mold investigations 
are SEM-EDS and pXRF analyses. The aim of this study is, first, to test the possibilities of ceramic casting mold analysis in detecting alloy traces and, second, to compare the data obtained with the earlier results on the Bohai period's compositions of copper alloys.

\section{Materials and Methods}

\subsection{Samples}

The total collection of ceramic molds contains 47 objects including 26 unbroken or damaged minimally molds and 21 fragmented ones. All objects are parts of small-sized two-sectioned, pouring-gated molds with multiple usages. No single completed mold consisting of coinciding sections, of halves, is recognized. In the collection, two-sided and one-sided casting molds are distinguished.

Two-sided rectangular-shaped molds are designed for the casting of semispherical buttons with a loop at the backside. The unbroken and fragmentary sections of these molds are most representative in the collection (Figure 2e-g). Semispherical looped buttons were the most common kind of bronzes spread over the vast territories of eastern Eurasia and, in particular, at the southern Russian Far East within broad chronological frames from the end of 2nd mil BC to the end of 1st mil AD. They occurred at some Bohai State period sites [11] (p. 72).

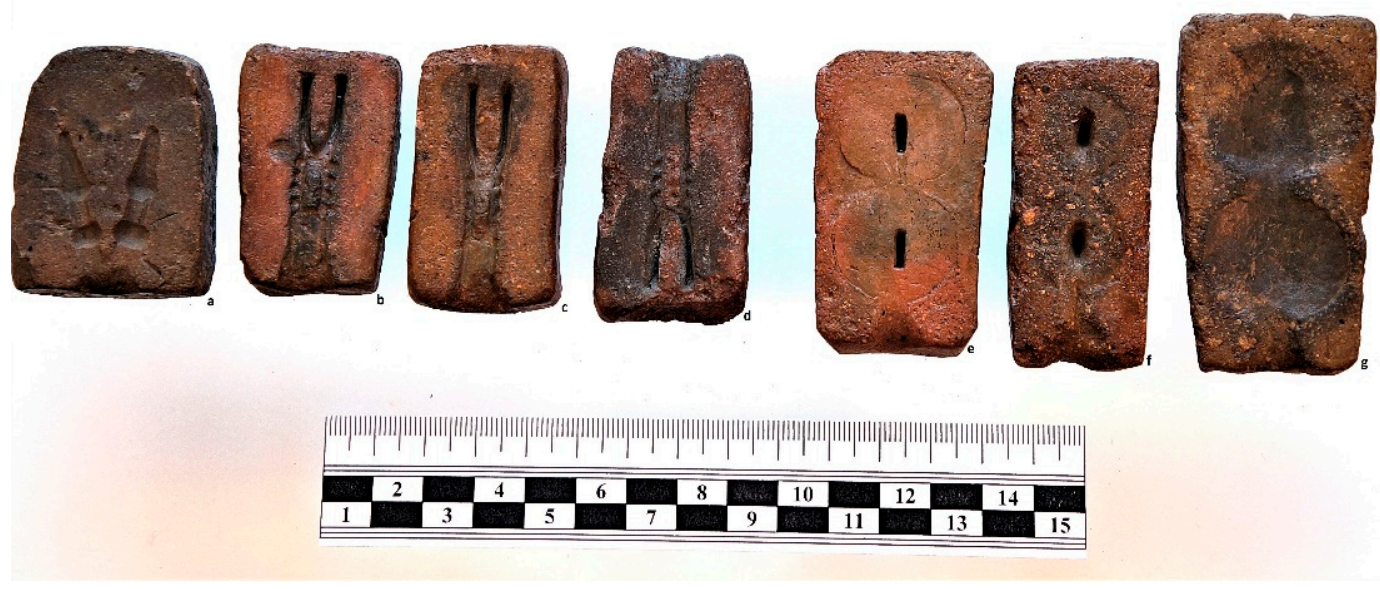

Figure 2. Kruglaya Dolina site. Ceramic casting molds for (a-d) "two-tailed" objects and (e-g) semispherical buttons. Completed items with surface glue transparent coverings.

One-sided rectangular-shaped molds are designed for the casting artifacts determined preliminary as "two-tailed" objects, likely pendants. Unbroken and fragmentary sections of these molds with entirely or partially preserved casting cavities comprise the representative series (Figures $2 a-d$ and $3 a-c$ ). A single bronze item quite identical to "two-tailed" pendantlike castings was found earlier at the destroyed archaeological site in northeastern Primorye, dated preliminary to the Bohai State period [11] (p. 72). A one-sided rectangular-shaped mold designed for the casting of a "three-tailed" object, likely a pendant, is represented by a single example of an unbroken section with a preserved casting cavity (Figure 3g).

One-sided square-shaped and rounded-shaped molds are designed for the casting of rosette-like objects, supposedly ornaments (Figure $3 \mathrm{~d}-\mathrm{f}$ ). They make up a series of six examples. The bronzes similar to rosette-shaped ornaments are not known at archaeological sites of the southern Russian Far East. 

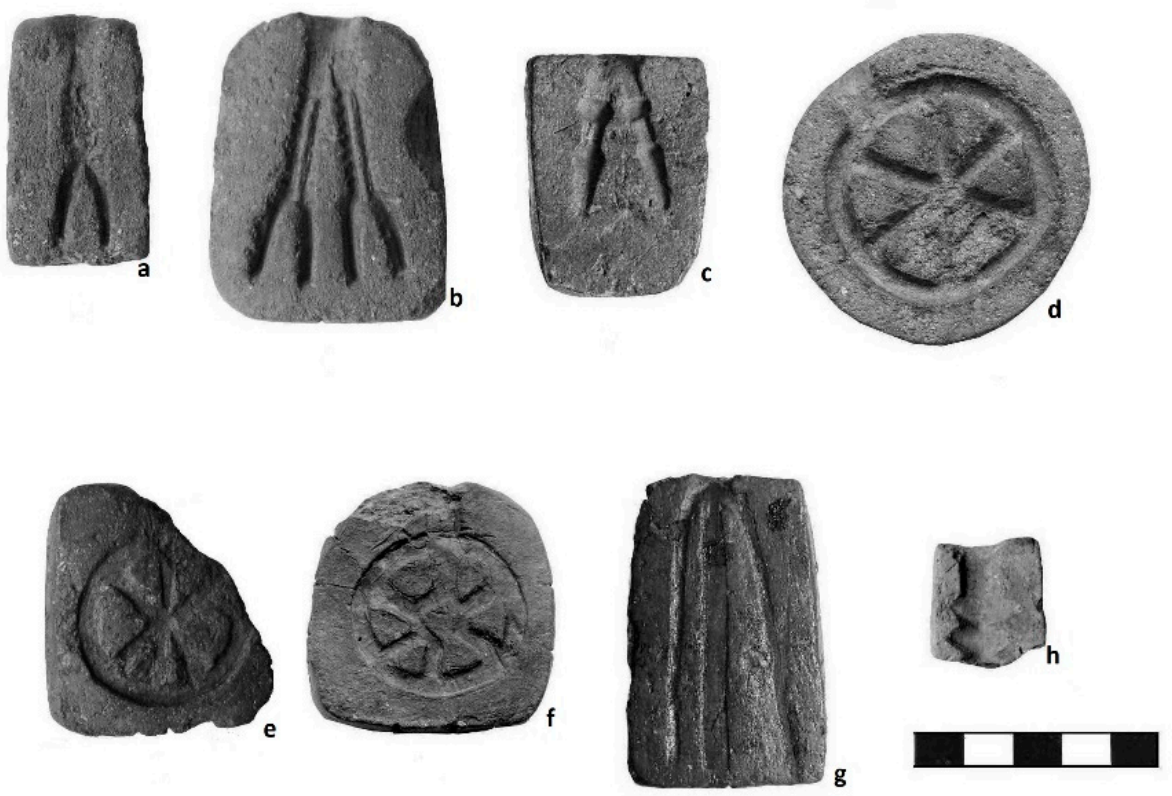

Figure 3. Kruglaya Dolina site. (a-h) Ceramic casting molds. Completed and fragmented items.

In some cases, the mold's fragment with the casting cavity is too small for correctly recognizing a bronze artifact image and the type of mold-two-sided or one-sided (Figure 3h). Besides the examples of molds with entirely and partially preserved casting cavities, a few examples of rectangular-shaped and square-shaped flat-surfaced lids, or covers, are presented in the collection. They functioned as the halves of one-sided molds.

The casting mold surfaces have yellowish, light-orange, and light-brownish colors. Most of the mold examples have dark-grey or black working surfaces, including a casting cavity zone and a pouring gate zone. This feature may be explained by the saturation of a porous ceramic material with carbon matter during the casting process. Some lids of single-sided molds have dark-colored zones at one of the flat surfaces. The series of molds, mostly fragmented, have clear non-carbonized surfaces of light colors. Supposedly, this indicates that the molds were produced but, for some reason, had not been used for the casting. Not a single mold example has any visible traces of metal at the surface of the casting zone (cavity and pouring gate).

It is important to note that, after the excavations in the early 1970s, ceramic molds were cleaned and washed, and all well-preserved unbroken examples were covered by a thin layer of transparent glue substance for better conservation and a more attractive external image. At that time, the usual practice was after-excavation processing of some artifact categories. Pieces of medical bandages were stuck on the molds' flat sides for artifact individual tagging. Later, the band-aid tags were removed, but their micro-particles remained at the ceramic surfaces. The presence of transparent glue slips and bandage traces was considered while the samples had been selected for physicochemical examination.

A total of 34 samples was selected for examination, including 13 unbroken and minimally damaged artifacts, and 21 fragmentary molds. All objects were designed with serial numbers 1-34 (Table 1). Taking into account the uniqueness of a ceramic casting mold assemblage from the Kruglaya Dolina site, an important task was to minimize the destruction or any changes to the objects targeted for analytical examination. 
Table 1. List of the objects sampled for the PXRF and SEM-EDS examination. Kruglaya Dolina site.

\begin{tabular}{|c|c|c|c|c|c|c|}
\hline Object Number & Kind of Object & Bronze Casting Type & Glue Covering & $\begin{array}{l}\text { Carbonization of } \\
\text { Working Surface }\end{array}$ & $\begin{array}{l}\text { SEM-EDS } \\
\text { Examined }\end{array}$ & pXRF Examined \\
\hline 1 & Mold's lid section (unbroken) & Not identified & + & + & - & + \\
\hline 2 & Casting section of two-sided mold & Semi-spherical looped button & + & + & - & + \\
\hline 3 & Casting section of one-sided mold & "two-tailed" object (pendant?) & + & + & - & + \\
\hline 4 & Casting section of two-sided mold & Semi-spherical looped button & + & + & - & + \\
\hline 5 & Casting section of two-sided mold & Semi-spherical looped button & + & + & - & + \\
\hline 7 & Casting section of one-sided mold & $\begin{array}{l}\text { "Two-tailed" pair objects } \\
\text { (pendants?) }\end{array}$ & + & + & - & + \\
\hline 8 & $\begin{array}{l}\text { Casting section of one-sided } \\
\text { mold(fragment) }\end{array}$ & $\begin{array}{l}\text { “Two-tailed" pair objects } \\
\text { (pendants?) }\end{array}$ & + & + & - & + \\
\hline 9 & Casting section of one-sided mold & “Three-tailed" object (pendant?) & + & + & - & + \\
\hline 10 & Casting section of one-sided mold & $\begin{array}{l}\text { Rosette-shaped object } \\
\text { (ornament?) }\end{array}$ & + & + & - & + \\
\hline 12 & Casting section of the mold(fragment) & Not identified & - & + & - & + \\
\hline 13 & Casting section of the mold (fragment) & Ornament? & - & + & + & + \\
\hline 14 & Casting section of the mold (fragment) & Not identified & - & - & - & + \\
\hline 15 & $\begin{array}{l}\text { Casting section of two-sided mold } \\
\text { (fragment) }\end{array}$ & Semi-spherical looped button & - & + & + & + \\
\hline 16 & $\begin{array}{l}\text { Casting section of two-sided mold } \\
\text { (fragment) }\end{array}$ & Semi-spherical looped button & - & - & + & + \\
\hline 17 & Casting section of the mold (fragment) & Not identified & - & - & + & + \\
\hline 18 & $\begin{array}{l}\text { Casting section of two-sided mold } \\
\text { (fragment) }\end{array}$ & Semi-spherical looped button & - & + & + & + \\
\hline 19 & $\begin{array}{l}\text { Casting section of one-sided mold } \\
\text { (fragment) }\end{array}$ & “Two-tailed” object (pendant?) & - & + & - & + \\
\hline
\end{tabular}


Table 1. Cont.

\begin{tabular}{|c|c|c|c|c|c|c|}
\hline Object Number & Kind of Object & Bronze Casting Type & Glue Covering & $\begin{array}{l}\text { Carbonization of } \\
\text { Working Surface }\end{array}$ & $\begin{array}{l}\text { SEM-EDS } \\
\text { Examined }\end{array}$ & pXRF Examined \\
\hline 20 & $\begin{array}{l}\text { Casting section of two-sided mold } \\
\text { (fragment) }\end{array}$ & Semi-spherical looped button & - & + & + & + \\
\hline 21 & $\begin{array}{l}\text { Casting section of one-sided mold } \\
\text { (fragment) }\end{array}$ & “Two-tailed" object (pendant?) & - & + & + & + \\
\hline 22 & $\begin{array}{l}\text { Casting section of two-sided mold } \\
\text { (fragment) }\end{array}$ & Semi-spherical looped button & - & - & + & + \\
\hline 23 & $\begin{array}{l}\text { Casting section of one-sided mold } \\
\text { (fragment) }\end{array}$ & Semi-spherical looped button? & - & + & + & + \\
\hline 24 & $\begin{array}{l}\text { Casting section of two-sided mold } \\
\text { (fragment) }\end{array}$ & Semi-spherical looped button & - & + & - & + \\
\hline 25 & Mold's lid section (fragment) & Not identified & - & + & + & + \\
\hline 26 & Mold's lid section & Not identified & - & - & + & + \\
\hline 27 & $\begin{array}{l}\text { Casting section of two-sided mold } \\
\text { (fragment) }\end{array}$ & Semi-spherical looped button & - & - & - & + \\
\hline 29 & $\begin{array}{l}\text { Casting section of one-sided mold } \\
\text { (fragment) }\end{array}$ & “Two-tailed” object (pendant?) & - & + & - & + \\
\hline 30 & Casting section of two-sided mold & Semi-spherical looped button & + & + & - & + \\
\hline 31 & $\begin{array}{l}\text { Casting section of two-sided mold } \\
\text { (fragment) }\end{array}$ & Semi-spherical looped button? & - & + & + & + \\
\hline 32 & Casting section of one-sided mold & “Two-tailed" object (pendant?) & + & + & - & + \\
\hline 33 & $\begin{array}{l}\text { Casting section of two-sided mold } \\
\text { (fragment) }\end{array}$ & Semi-spherical looped button & - & + & + & + \\
\hline 34 & $\begin{array}{l}\text { Casting section of two-sided mold } \\
\text { (fragment) }\end{array}$ & Semi-spherical looped button & - & + & + & + \\
\hline
\end{tabular}




\subsection{Research Methods}

For the characteristic of the ceramic fabrics from the mold, this study uses binocular microscopy of polished cross sections, SEM method, and the water absorption index. Binocular microscopy of polished cross sections allows us to observe the main textural features, to determine general types and distribution patterns of non-plastic inclusions, and to approximate the amount and shapes of the voids $[14,15]$. This research includes the study of 12 fractured mold samples. A ceramic fabric microstructure analysis with SEM allows us to estimate the firing temperature based on the degree of sintering or vitrification of clay particles [16,17]. Two objects with "clear" non-carbonized surfaces (number 17 and 26) were analyzed under SEM. Measuring the water absorption (WA) index is helpful for the relative evaluation of a ceramic fabric's density, porosity, and firing quality [16] (pp. 125-130); [17] (pp. 350-354). The WA index was measured for 12 samples series examined with binocular microscopy of the polished sections.

The $\mathrm{pXRF}$ and SEM-EDS were proposed to determine the chemical composition of the ceramic mold surfaces and to detect the elements that might be interpreted as alloy traces. This methodology has published results after identifying traces of a copper-alloy chemical composition on the surface of experimental ceramic casting molds. As has been proven, elemental compositions of alloy traces that may be recognized at the molds are not analogous to the original alloy compositions. The elements $\mathrm{Pb}, \mathrm{Sn}, \mathrm{Cu}$, and $\mathrm{Zn}$, which are the most important as probable alloy components, could be determined at ceramic mold surfaces with varying degrees of accuracy. $\mathrm{Pb}$ is detected the quickest because this element forms a stable chemical compound with silica (Si) as a primary clay and ceramic composition component. On the contrary, the chemical compounds of Sn and $\mathrm{Cu}$ with $\mathrm{Si}$ are much more unstable and volatile. Traces of these elements on the ceramic surface are weaker and more difficult to diagnose. The identification of $\mathrm{Zn}$ also has specific difficulties [9]. These points were taken into account for understanding and interpreting the pXRF and SEM-EDS research data.

The first step of this research involved using the XRF method and applying an Olympus Delta Professional DP 4000 portable X-ray fluorescence analyzer (pXRF) (Waltham, MA, USA). The method provides a possibility to analyze surface areas up to $10-20 \mathrm{~mm}$, giving an average quantitative indication of the elemental composition with limitations on the number and accuracy of the elements to be determined. The pXRF analyzer was calibrated with the Olympus Analytical Instrument 316 calibration check reference coin. The threshold for the presence of elements in this study was chosen to be $0.02 \%$. In this study, the data obtained by the pXRF analyzer complemented the scanning electron microscopy with energy dispersive spectrometer (SEM-EDS) data by analyzing surfaces with glue coatings. The pXRF was chosen as a nondestructive method that is non-sensitive for organic matter since SEM-EDS is inconvenient for application in objects coated with glue. Removal of the glue results in partial loss of the outer layer of the mold, causing undesirable and irreversible loss of traces of the alloying elements. In the current study, glue removal was not attempted. The "working" surfaces of the molds, in particular casting cavities and pouring gates areas, were of primary interest for the determination of elemental compositions. The "non-working" surfaces of the molds were also tested for comparison.

The SEM-EDS method was used to determine the chemical compositions and microstructures of ceramic substance at the molds' working surfaces, including casting cavities and pouring gates. Twelve fragmented mold halves with partially preserved working surfaces and one unbroken lid were selected for the examination (Table 1). All objects were free of glue coating and visible traces of band-aid tags at the surfaces. Most of the examined objects have traces of carbonization (Figure 4). In most cases, the object sizes allow for recognition of the kind of casted bronze image. The SEM-EDS equipment used for the examination was a ZEISS scanning electron microscope EVO-40 (Oberkochen, Germany). The SEM device was fitted with the energy dispersive spectrometer (EDS) of the 
Oxford Instruments INCA-x sight. The acceleration voltage was kept at $20 \mathrm{kV}$; the working distance was kept at 7.0-17.0 mm; and the magnifications were $300 \times, 500 \times$, and $1000 \times$.

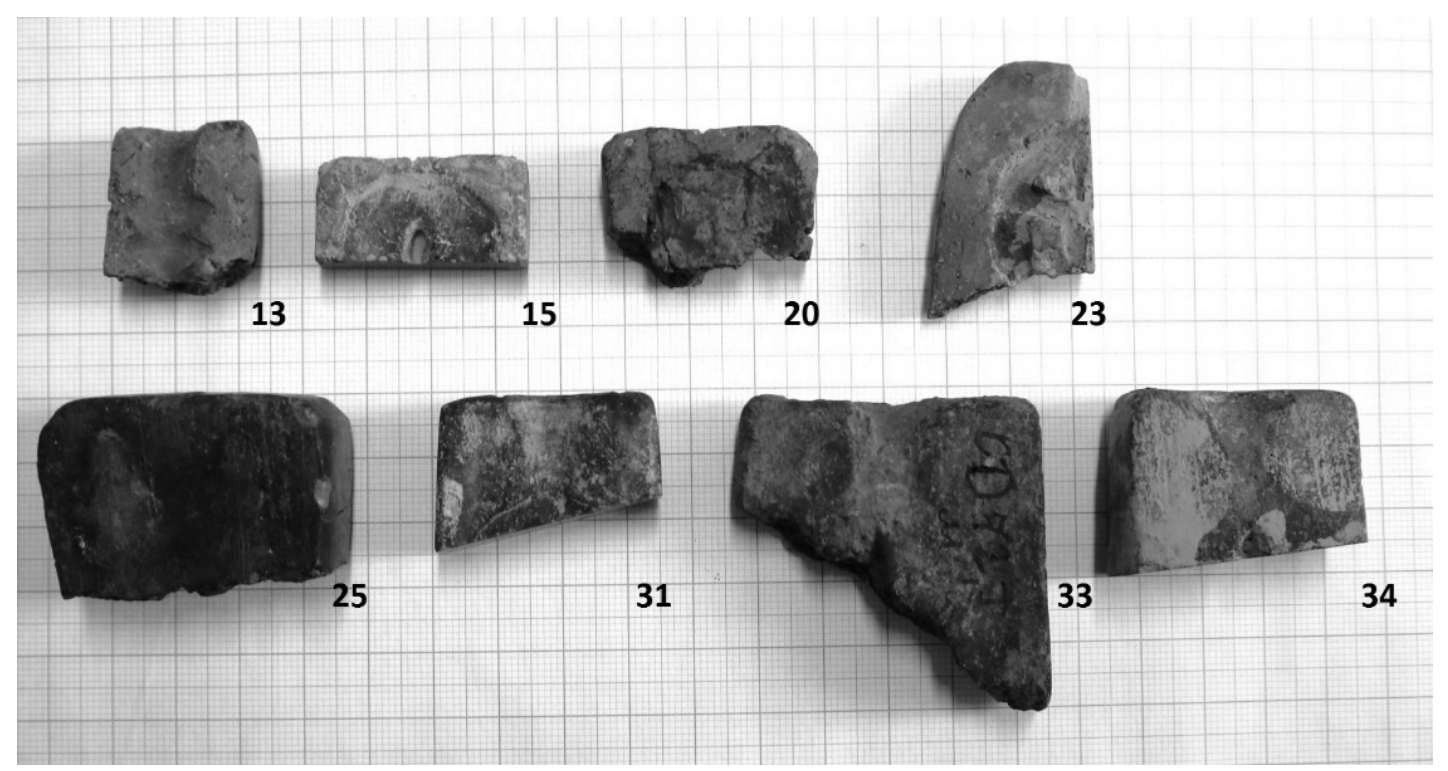

Figure 4. Kruglaya Dolina site. SEM-EDS examined objects (numbered) with carbonized cavities' surfaces.

Using SEM-EDS, the surfaces of (ceramic) molds in the zones of probable contact with metal, i.e., in the casting zone and in the pouring gate zone, were studied. The number of test sites for one object was from 1 to 3 . The number of points for shooting EDS spectra on one site was from 11 to 26 . The total number of spectra for one object was from 11 to 61. Taking into account that the EDS method allows for determining the content of chemical elements (in \% of the weight composition) at a semi-quantitative level, values from $1.0 \%$ and higher were taken into account for the correctness of observations and subsequent interpretations.

\section{Results}

\subsection{Results of Ceramic Fabric Examination}

Based on visual observations, the researchers noted that ceramic casting molds from the Kruglaya Dolina site were made of clay with a large amount of sand temper [11] (p. 71). The current data precisely suggest this conclusion. Based on the binocular microscopy of polished cross sections, two kinds of ceramics fabric are distinguished.

A ceramic fabric of the first kind is determined as a "thin-textured" one (Figure 5a). This fabric contains a high amount of non-plastic mineral inclusions $\leq 0.2 \mathrm{~mm}$ in size and around $10-15 \%$ of non-plastic inclusions $\leq 1.0 \mathrm{~mm}$ in size. The inclusions recognized as quartz and feldspar seem to be the natural temper of the raw clay material. Very small-sized voids are of a roundish shape. This fabric was interpreted probably as natural clay and was cleaned carefully but not tempered with intentional adds. Thin-textured fabric is characteristic for small series of molds.

A ceramic fabric of the second kind is determined as a "coarse-textured" one containing non-plastic mineral inclusions of two size ranges. The smallest inclusions $\leq 0.2 \mathrm{~mm}$ in size are abundant as in the ceramic fabric of the first group (Figure $5 b$ ). Inclusions $0.5-2.5 \mathrm{~mm}$ in size composing from $10-15 \%$ to $25-30 \%$ of the fabric's volume represent mainly quartz and feldspar grains, and granite rock grains. Sometimes, sedimentary rocks grains and hematite rock grains are present. These coarse inclusions showing relatively even distributions in the fabric matter are interpreted supposedly as intendedly added temper. Coarse-textured fabric is characteristic for most of the examined molds. 


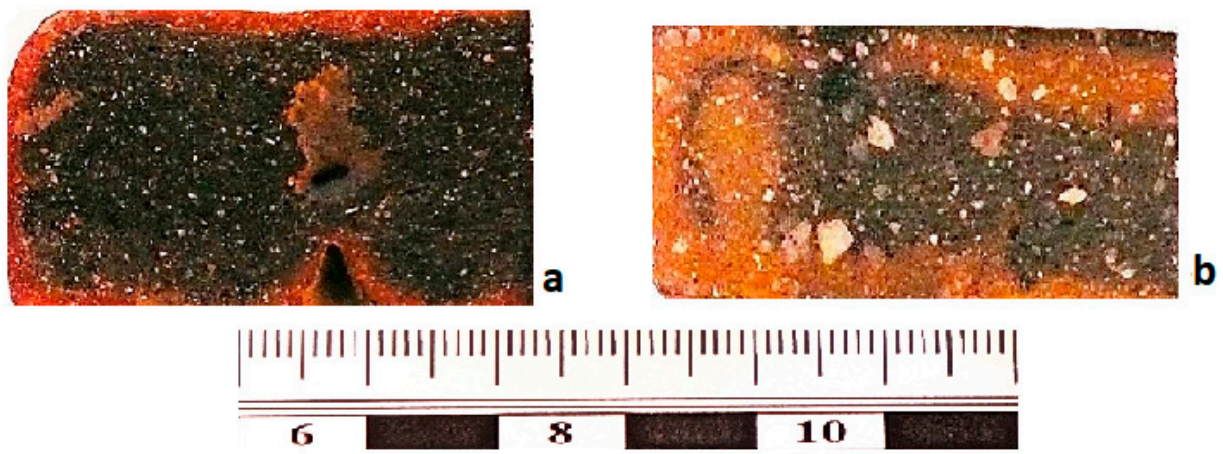

Figure 5. Kruglaya Dolina site. Polished samples of (a) thin-textured ceramic fabric and (b) coarsetextured ceramic fabric.

The water absorption indexes measured for 10 samples representing both textural groups are in the interval of 10.2-14.5\%. These values corresponding to moderate rates of water absorption indicate a relatively porous but not highly dense ceramic body [16] (pp. 128-130). SEM examination of the microstructure of the non-carbonized cavity surface (object number 17) and non-carbonized lid's surface (object number 26) showed the absence of evidence of even initial vitrification (Figure 6a,b). As is known, the vitrification processes in the clay particles of ceramic matter begin in the interval of $800-850{ }^{\circ} \mathrm{C}$ in an oxidizing firing atmosphere $[18,19]$. Therefore, the test results suppose that casting molds were fired at temperatures not higher than $800{ }^{\circ} \mathrm{C}$.
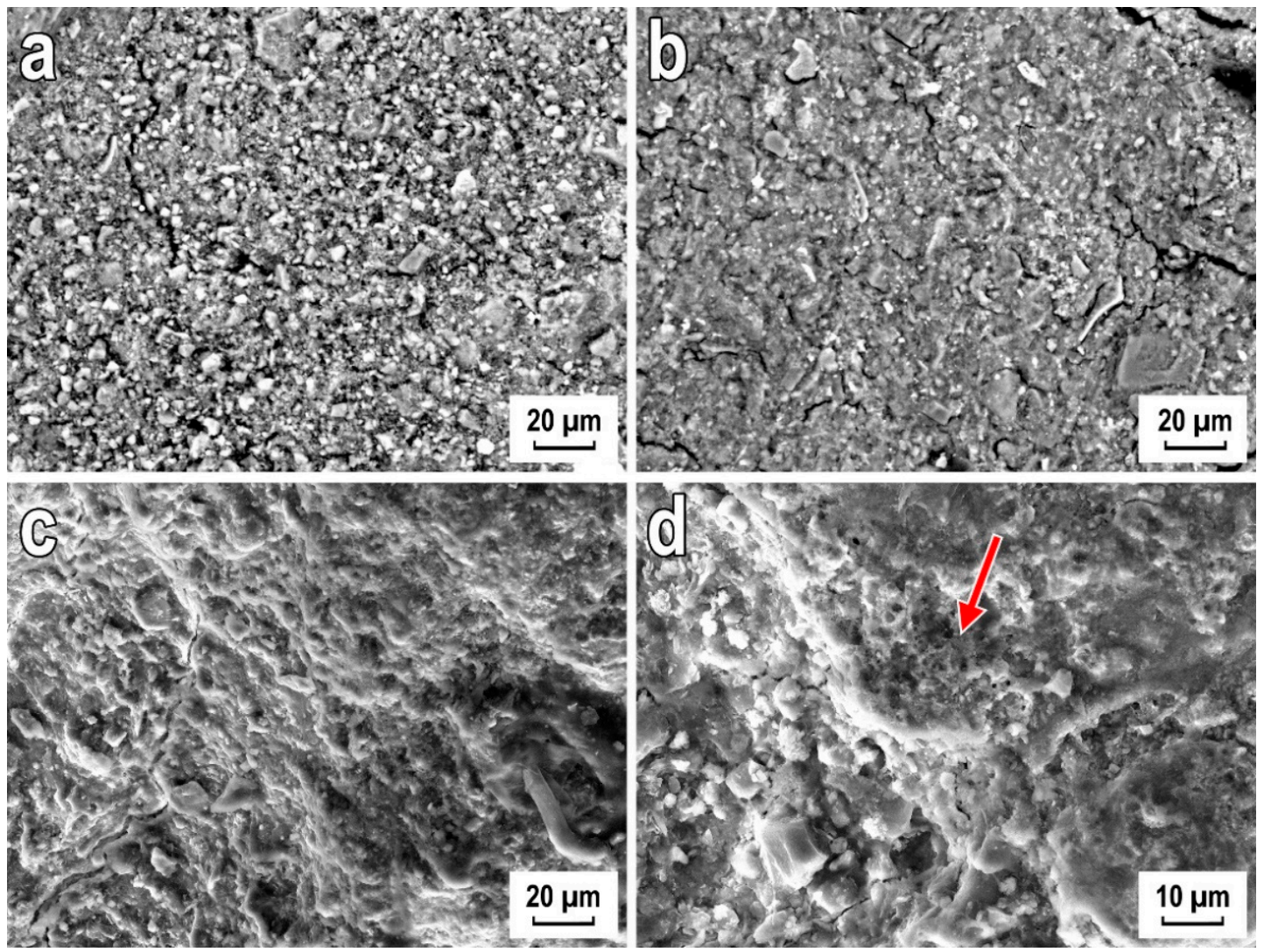

Figure 6. Kruglaya Dolina site. Selected SEM images of examined objects. (a) Object number 17, non-carbonized; cavity surface microstructure. (b) Object number 26, non-carbonized; surface microstructure. (c) Object number 33, carbonized; cavity surface microstructure with clay particles sintering evidence. (d) Object number 33, carbonized; cavity surface microstructure with the area of bubbling (arrow marked). 
These properties caused by firing technology are somewhat different from the properties of ceramic wares unearthed at many Bohai State period sites in the Primorye region. The ceramic body of the pots produced with pottery wheel and fired in a reduced regime often shows evidence of vitrification, and the water absorption index is mostly within the limits of $5.0-10.0 \%$. Supposedly, Bohai pottery firing temperatures were around $850{ }^{\circ} \mathrm{C}$ or above [20]. In this connection, it is interesting to note that ceramic casting molds from Chinese sites of the Eastern Zhou period (770-256 BC) were fired at temperatures lower than the firing temperature range of ordinary ceramic wares: $800-1000{ }^{\circ} \mathrm{C}$ [4]. There are other known cases of old ceramic casting molds fired at relatively low temperatures [8,21].

In general, the fabric of casting molds from the Kruglaya Dolina site represents nonvitrified ceramic material with a porous texture. Concerning ceramic molds, this texture facilitates the penetration of a hot liquid alloy's micro-particles into the surface layer during the casting process [10].

\subsection{Results of $p X R F$ Screening Analysis Examination of Mold Surfaces}

The results of pXRF examination of the molds' surfaces are presented in Table 2, and the data of the metal elements assumed to be of the alloy composition $(\mathrm{Pb}, \mathrm{Cu}, \mathrm{Sn}$, and $\mathrm{Zn})$ are visualized in Figure 7.
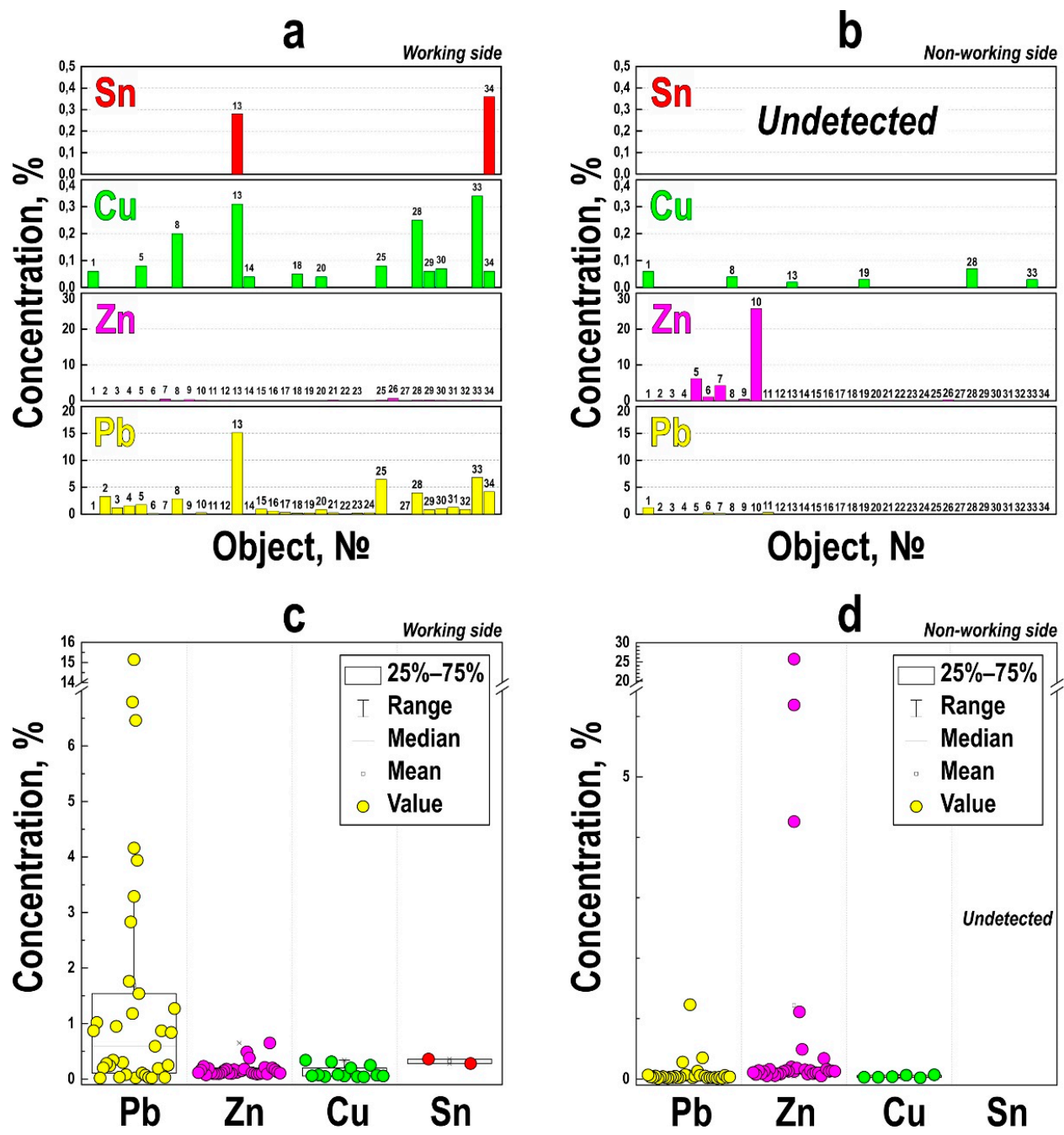

Figure 7. Kruglaya Dolina site. Results of the pXRF screening analysis examination of molds for $(\mathbf{a}, \mathbf{c})$ the working and $(\mathbf{b}, \mathbf{d})$ the non-working surfaces. 
Table 2. Kruglaya Dolina site. Results of the pXRF screening analysis examination of molds on the working and non-working surfaces.

\begin{tabular}{|c|c|c|c|c|c|c|c|c|c|c|c|c|c|c|c|c|c|}
\hline Object Number & $\overline{\mathrm{Si}}$ & $\mathrm{Fe}$ & $\mathrm{Al}$ & $P$ & $\mathrm{Ti}$ & $\mathrm{S}$ & $\mathrm{Pb}$ & Mn & $\mathrm{Zr}$ & $\mathrm{Zn}$ & $\mathrm{Cu}$ & $\mathrm{Ni}$ & $\mathrm{V}$ & $\mathbf{B i}$ & $\mathrm{Nb}$ & $\mathrm{Cr}$ & Sn \\
\hline \multicolumn{18}{|c|}{ Working Side } \\
\hline 1 & $\begin{array}{l}43.87 \\
\pm 0.18\end{array}$ & $\begin{array}{c}28.87 \\
\pm 0.16\end{array}$ & $\begin{array}{c}20.84 \\
\pm 0.22\end{array}$ & $\begin{array}{c}1.78 \\
\pm 0.03\end{array}$ & $\begin{array}{c}1.73 \\
\pm 0.11\end{array}$ & $\begin{array}{c}1.65 \\
\pm 0.03\end{array}$ & $\begin{array}{c}0.02 \\
\pm 0.005\end{array}$ & $\begin{array}{c}0.79 \\
\pm 0.04\end{array}$ & $\begin{array}{c}0.14 \\
\pm 0.004\end{array}$ & $\begin{array}{c}0.14 \\
\pm 0.01\end{array}$ & $\begin{array}{c}0.06 \\
\pm 0.009\end{array}$ & - & $\begin{array}{c}0.11 \\
\pm 0.03\end{array}$ & - & - & - & - \\
\hline 2 & $\begin{array}{l}47.49 \\
\pm 0.18\end{array}$ & $\begin{array}{l}25.66 \\
\pm 0.16\end{array}$ & $\begin{array}{l}17.81 \\
\pm 0.21\end{array}$ & $\begin{array}{c}2.24 \\
\pm 0.03\end{array}$ & $\begin{array}{c}2.55 \\
\pm 0.13\end{array}$ & - & $\begin{array}{c}3.29 \\
\pm 0.04\end{array}$ & $\begin{array}{c}0.55 \\
\pm 0.04\end{array}$ & $\begin{array}{c}0.24 \\
\pm 0.006\end{array}$ & $\begin{array}{c}0.12 \\
\pm 0.01\end{array}$ & - & $\begin{array}{c}0.05 \\
\pm 0.01\end{array}$ & - & - & - & - & - \\
\hline 3 & $\begin{array}{l}46.94 \\
\pm 0.19\end{array}$ & $\begin{array}{l}25.66 \\
\pm 0.17\end{array}$ & $\begin{array}{c}18.67 \\
\pm 0.22\end{array}$ & $\begin{array}{c}2.29 \\
\pm 0.04\end{array}$ & $\begin{array}{c}2.69 \\
\pm 0.14\end{array}$ & $\begin{array}{c}1.85 \\
\pm 0.03\end{array}$ & $\begin{array}{c}1.18 \\
\pm 0.02\end{array}$ & $\begin{array}{c}0.06 \\
\pm 0.04\end{array}$ & $\begin{array}{c}0.28 \\
\pm 0.006\end{array}$ & $\begin{array}{c}0.15 \\
\pm 0.01\end{array}$ & - & $\begin{array}{c}0.06 \\
\pm 0.02\end{array}$ & $\begin{array}{c}0.17 \\
\pm 0.04\end{array}$ & - & - & - & - \\
\hline 4 & $\begin{array}{c}47.15 \\
\pm 0.2\end{array}$ & $\begin{array}{c}28.81 \\
\pm 0.18\end{array}$ & $\begin{array}{c}17.04 \\
\pm 0.24\end{array}$ & $\begin{array}{c}1.78 \\
\pm 0.04\end{array}$ & $\begin{array}{c}2.66 \\
\pm 0.14\end{array}$ & - & $\begin{array}{c}1.54 \\
\pm 0.03\end{array}$ & $\begin{array}{c}0.35 \\
\pm 0.03\end{array}$ & $\begin{array}{c}0.34 \\
\pm 0.007\end{array}$ & $\begin{array}{c}0.17 \\
\pm 0.01\end{array}$ & - & - & $\begin{array}{c}0.16 \\
\pm 0.04\end{array}$ & - & - & - & - \\
\hline 5 & $\begin{array}{c}51.16 \\
\pm 0.2\end{array}$ & $\begin{array}{c}26.11 \\
\pm 0.17\end{array}$ & $\begin{array}{c}15.98 \\
\pm 0.21\end{array}$ & $\begin{array}{c}1.09 \\
\pm 0.03\end{array}$ & $\begin{array}{c}2.69 \\
\pm 0.15\end{array}$ & - & $\begin{array}{c}1.76 \\
\pm 0.03\end{array}$ & $\begin{array}{c}0.39 \\
\pm 0.04\end{array}$ & $\begin{array}{c}0.33 \\
\pm 0.007\end{array}$ & $\begin{array}{c}0.18 \\
\pm 0.01\end{array}$ & $\begin{array}{c}0.08 \\
\pm 0.01\end{array}$ & $\begin{array}{c}0.07 \\
\pm 0.02\end{array}$ & $\begin{array}{c}0.16 \\
\pm 0.04\end{array}$ & - & - & - & - \\
\hline 6 & $\begin{array}{c}44.84 \\
\pm 0.19\end{array}$ & $\begin{array}{l}27.75 \\
\pm 0.18\end{array}$ & $\begin{array}{c}19.63 \\
\pm 0.23\end{array}$ & $\begin{array}{c}2.00 \\
\pm 0.03\end{array}$ & $\begin{array}{c}2.50 \\
\pm 0.14\end{array}$ & $\begin{array}{c}1.57 \\
\pm 0.03\end{array}$ & $\begin{array}{c}0.11 \\
\pm 0.009\end{array}$ & $\begin{array}{c}0.99 \\
\pm 0.05\end{array}$ & $\begin{array}{c}0.27 \\
\pm 0.006\end{array}$ & $\begin{array}{c}0.10 \\
\pm 0.01\end{array}$ & - & $\begin{array}{c}0.08 \\
\pm 0.02\end{array}$ & $\begin{array}{c}0.16 \\
\pm 0.04\end{array}$ & - & - & - & - \\
\hline 7 & $\begin{array}{c}34.30 \\
\pm 0.2\end{array}$ & $\begin{array}{l}42.20 \\
\pm 0.25\end{array}$ & $\begin{array}{l}15.33 \\
\pm 0.3\end{array}$ & $\begin{array}{c}1.33 \\
\pm 0.04\end{array}$ & $\begin{array}{c}3.54 \\
\pm 0.17\end{array}$ & $\begin{array}{c}1.59 \\
\pm 0.04\end{array}$ & $\begin{array}{c}0.08 \\
\pm 0.01\end{array}$ & $\begin{array}{c}0.55 \\
\pm 0.05\end{array}$ & $\begin{array}{c}0.59 \\
\pm 0.01\end{array}$ & $\begin{array}{c}0.49 \\
\pm 0.03\end{array}$ & - & - & - & - & - & $\begin{array}{c}0.17 \\
\pm 0.04\end{array}$ & - \\
\hline 8 & $\begin{array}{c}48.63 \\
\pm 0.17 \\
\end{array}$ & $\begin{array}{l}23.70 \\
\pm 0.15 \\
\end{array}$ & $\begin{array}{l}20.82 \\
\pm 0.2 \\
\end{array}$ & $\begin{array}{c}0.89 \\
\pm 0.02 \\
\end{array}$ & $\begin{array}{c}2.27 \\
\pm 0.12 \\
\end{array}$ & - & $\begin{array}{c}2.83 \\
\pm 0.03 \\
\end{array}$ & $\begin{array}{c}0.29 \\
\pm 0.03 \\
\end{array}$ & $\begin{array}{c}0.22 \\
\pm 0.005 \\
\end{array}$ & $\begin{array}{c}0.11 \\
\pm 0.008 \\
\end{array}$ & $\begin{array}{c}0.20 \\
\pm 0.01 \\
\end{array}$ & $\begin{array}{c}0.04 \\
\pm 0.01 \\
\end{array}$ & - & - & - & - & - \\
\hline 10 & $\begin{array}{l}45.04 \\
\pm 0.18\end{array}$ & $\begin{array}{l}29.75 \\
\pm 0.17\end{array}$ & $\begin{array}{c}18.80 \\
\pm 0.23\end{array}$ & $\begin{array}{c}1.38 \\
\pm 0.03\end{array}$ & $\begin{array}{c}2.54 \\
\pm 0.12\end{array}$ & $\begin{array}{c}0.96 \\
\pm 0.03\end{array}$ & $\begin{array}{c}0.30 \\
\pm 0.01\end{array}$ & $\begin{array}{c}0.56 \\
\pm 0.04\end{array}$ & $\begin{array}{c}0.24 \\
\pm 0.005\end{array}$ & $\begin{array}{c}0.18 \\
\pm 0.01\end{array}$ & - & $\begin{array}{c}0.09 \\
\pm 0.02\end{array}$ & $\begin{array}{c}0.16 \\
\pm 0.04\end{array}$ & - & - & - & - \\
\hline 11 & $\begin{array}{l}44.83 \\
\pm 0.15\end{array}$ & $\begin{array}{l}23.03 \\
\pm 0.13 \\
\end{array}$ & $\begin{array}{l}22.95 \\
\pm 0.18\end{array}$ & $\begin{array}{c}4.87 \\
\pm 0.04 \\
\end{array}$ & $\begin{array}{l}2.21 \\
\pm 0.1 \\
\end{array}$ & $\begin{array}{c}1.12 \\
\pm 0.02 \\
\end{array}$ & $\begin{array}{c}0.03 \\
\pm 0.004 \\
\end{array}$ & $\begin{array}{c}0.47 \\
\pm 0.03 \\
\end{array}$ & $\begin{array}{c}0.16 \\
\pm 0.003 \\
\end{array}$ & $\begin{array}{c}0.11 \\
\pm 0.007 \\
\end{array}$ & - & $\begin{array}{c}0.06 \\
\pm 0.009 \\
\end{array}$ & $\begin{array}{c}0.16 \\
\pm 0.03 \\
\end{array}$ & - & $\begin{array}{c}0.01 \\
\pm 0.002 \\
\end{array}$ & - & - \\
\hline 12 & $\begin{array}{l}46.45 \\
\pm 0.2\end{array}$ & $\begin{array}{c}28.04 \\
\pm 0.19\end{array}$ & $\begin{array}{l}20.10 \\
\pm 0.22\end{array}$ & $\begin{array}{c}1.10 \\
\pm 0.03\end{array}$ & $\begin{array}{c}2.52 \\
\pm 0.15\end{array}$ & $\begin{array}{c}0.68 \\
\pm 0.03\end{array}$ & $\begin{array}{c}0.04 \\
\pm 0.007\end{array}$ & $\begin{array}{c}0.47 \\
\pm 0.04\end{array}$ & $\begin{array}{c}0.25 \\
\pm 0.006\end{array}$ & $\begin{array}{c}0.15 \\
\pm 0.01\end{array}$ & - & $\begin{array}{c}0.08 \\
\pm 0.02\end{array}$ & $\begin{array}{c}0.13 \\
\pm 0.04\end{array}$ & - & - & - & - \\
\hline 13 & $\begin{array}{c}40.95 \\
\pm 0.14\end{array}$ & $\begin{array}{c}16.15 \\
\pm 0.12\end{array}$ & $\begin{array}{c}19.47 \\
\pm 0.17\end{array}$ & $\begin{array}{c}5.54 \\
\pm 0.04\end{array}$ & $\begin{array}{l}1.62 \\
0.11\end{array}$ & - & $\begin{array}{l}15.14 \\
\pm 0.08\end{array}$ & $\begin{array}{c}0.33 \\
\pm 0.03\end{array}$ & $\begin{array}{c}0.24 \\
\pm 0.006\end{array}$ & $\begin{array}{c}0.10 \\
\pm 0.008\end{array}$ & $\begin{array}{c}0.31 \\
\pm 0.01\end{array}$ & - & $\begin{array}{c}0.15 \\
\pm 0.04\end{array}$ & - & - & - & $\begin{array}{c}0.28 \\
\pm 0.03\end{array}$ \\
\hline 14 & $\begin{array}{c}49.38 \\
\pm 0.18\end{array}$ & $\begin{array}{c}23.98 \\
\pm 0.15\end{array}$ & $\begin{array}{c}20.59 \\
\pm 0.2\end{array}$ & $\begin{array}{c}2.34 \\
\pm 0.03\end{array}$ & $\begin{array}{l} \pm 2.45 \\
\pm 0.12\end{array}$ & $\begin{array}{c}0.28 \\
\pm 0.02\end{array}$ & $\begin{array}{c}0.023 \\
\pm 0.004\end{array}$ & $\begin{array}{c}0.47 \\
\pm 0.03\end{array}$ & $\begin{array}{c}0.17 \\
\pm 0.004\end{array}$ & $\begin{array}{c}0.11 \\
\pm 0.009\end{array}$ & $\begin{array}{c}0.04 \\
\pm 0.008\end{array}$ & - & $\begin{array}{c}0.16 \\
\pm 0.04\end{array}$ & - & - & - & - \\
\hline 15 & $\begin{array}{c}51.84 \\
\pm 0.16\end{array}$ & $\begin{array}{c}19.17 \\
\pm 0.12\end{array}$ & $\begin{array}{c}22.19 \\
\pm 0.18\end{array}$ & $\begin{array}{c}3.08 \\
\pm 0.03\end{array}$ & $\begin{array}{l}2.08 \\
\pm 0.1\end{array}$ & - & $\begin{array}{c}0.95 \\
\pm 0.02\end{array}$ & $\begin{array}{c}0.24 \\
\pm 0.02\end{array}$ & $\begin{array}{c}0.17 \\
\pm 0.003\end{array}$ & $\begin{array}{c}0.09 \\
\pm 0.006\end{array}$ & - & - & $\begin{array}{c}0.19 \\
\pm 0.03\end{array}$ & - & - & - & - \\
\hline
\end{tabular}


Table 2. Cont.

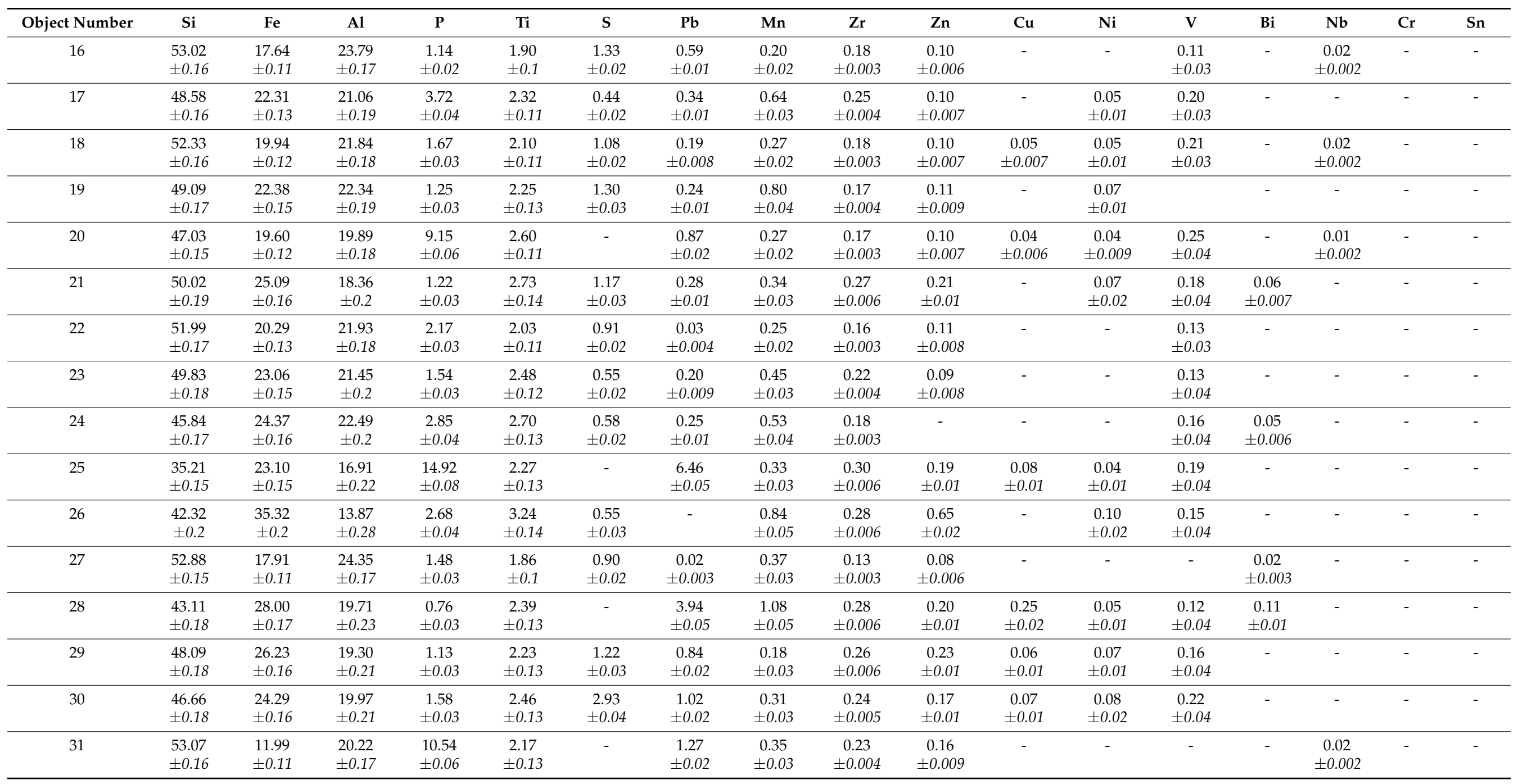


Table 2. Cont.

\begin{tabular}{|c|c|c|c|c|c|c|c|c|c|c|c|c|c|c|c|c|c|}
\hline Object Number & Si & $\mathrm{Fe}$ & Al & $\mathbf{P}$ & $\mathrm{Ti}$ & $\mathrm{S}$ & $\mathrm{Pb}$ & Mn & $\mathrm{Zr}$ & Zn & $\mathrm{Cu}$ & $\mathbf{N i}$ & V & Bi & $\mathrm{Nb}$ & $\mathrm{Cr}$ & Sn \\
\hline 32 & $\begin{array}{l}46.46 \\
\pm 0.2\end{array}$ & $\begin{array}{l}27.21 \\
\pm 0.18\end{array}$ & $\begin{array}{c}19.73 \\
\pm 0.23\end{array}$ & $\begin{array}{c}1.14 \\
\pm 0.03\end{array}$ & $\begin{array}{c}1.86 \\
\pm 0.13\end{array}$ & $\begin{array}{c}1.42 \\
\pm 0.03\end{array}$ & $\begin{array}{c}0.87 \\
\pm 0.02 \\
\end{array}$ & $\begin{array}{c}0.69 \\
\pm 0.05\end{array}$ & $\begin{array}{c}0.26 \\
\pm 0.006 \\
\end{array}$ & $\begin{array}{c}0.13 \\
\pm 0.01 \\
\end{array}$ & - & $\begin{array}{c}0.11 \\
\pm 0.02 \\
\end{array}$ & $\begin{array}{c}0.12 \\
\pm 0.04\end{array}$ & - & - & $\begin{array}{c}0.11 \\
\pm 0.03\end{array}$ & - \\
\hline 33 & $\begin{array}{c}42.9 \\
\pm 0.14\end{array}$ & $\begin{array}{c}20.28 \\
\pm 0.12\end{array}$ & $\begin{array}{l}20.57 \\
\pm 0.18\end{array}$ & $\begin{array}{c}6.30 \\
\pm 0.05\end{array}$ & $\begin{array}{c}1.99 \\
\pm 0.1\end{array}$ & - & $\begin{array}{c}6.79 \\
\pm 0.05\end{array}$ & $\begin{array}{c}0.30 \\
\pm 0.02\end{array}$ & $\begin{array}{c}0.24 \\
\pm 0.005\end{array}$ & $\begin{array}{c}0.17 \\
\pm 0.008\end{array}$ & $\begin{array}{c}0.34 \\
\pm 0.01\end{array}$ & $\begin{array}{c}0.04 \\
\pm 0.009\end{array}$ & $\begin{array}{c}0.13 \\
\pm 0.03\end{array}$ & - & $\begin{array}{c}0.02 \\
\pm 0.003\end{array}$ & - & - \\
\hline 34 & $\begin{array}{c}48.51 \\
\pm 0.16 \\
\end{array}$ & $\begin{array}{c}19.78 \\
\pm 0.14\end{array}$ & $\begin{array}{l}19.90 \\
\pm 0.18\end{array}$ & $\begin{array}{c}4.68 \\
\pm 0.04 \\
\end{array}$ & $\begin{array}{c}2.10 \\
\pm 0.12 \\
\end{array}$ & - & $\begin{array}{c}4.16 \\
\pm 0.04 \\
\end{array}$ & $\begin{array}{c}0.21 \\
\pm 0.03 \\
\end{array}$ & $\begin{array}{c}0.23 \\
\pm 0.005 \\
\end{array}$ & $\begin{array}{c}0.11 \\
\pm 0.008 \\
\end{array}$ & $\begin{array}{c}0.06 \\
\pm 0.009 \\
\end{array}$ & $\begin{array}{c}0.04 \\
\pm 0.01 \\
\end{array}$ & $\begin{array}{c}0.14 \\
\pm 0.04 \\
\end{array}$ & $\begin{array}{c}0.09 \\
\pm 0.01 \\
\end{array}$ & - & - & $\begin{array}{c}0.36 \\
\pm 0.02 \\
\end{array}$ \\
\hline \multicolumn{18}{|c|}{ Non-Working Side } \\
\hline 1 & $\begin{array}{c}42.34 \\
\pm 0.17\end{array}$ & $\begin{array}{c}30.22 \\
\pm 0.16\end{array}$ & $\begin{array}{c}19.97 \\
\pm 0.22\end{array}$ & $\begin{array}{c}2.25 \\
\pm 0.03 \\
\end{array}$ & $\begin{array}{l}1.74 \\
\pm 0.1\end{array}$ & $\begin{array}{c}1.62 \\
\pm 0.03 \\
\end{array}$ & $\begin{array}{c}1.23 \\
\pm 0.02 \\
\end{array}$ & $\begin{array}{c}0.24 \\
\pm 0.03 \\
\end{array}$ & $\begin{array}{c}0.15 \\
\pm 0.004 \\
\end{array}$ & $\begin{array}{c}0.12 \\
\pm 0.009 \\
\end{array}$ & $\begin{array}{c}0.06 \\
\pm 0.05 \\
\end{array}$ & $\begin{array}{c}0.05 \\
\pm 0.01 \\
\end{array}$ & - & - & - & - & - \\
\hline 2 & $\begin{array}{l}46.34 \\
\pm 0.18\end{array}$ & $\begin{array}{c}26.55 \\
\pm 0.16\end{array}$ & $\begin{array}{l}18.89 \\
\pm 0.23\end{array}$ & $\begin{array}{c}2.08 \\
\pm 0.03\end{array}$ & $\begin{array}{c}2.45 \\
\pm 0.12\end{array}$ & $\begin{array}{c}1.77 \\
\pm 0.03\end{array}$ & $\begin{array}{c}0.06 \\
\pm 0.006 \\
\end{array}$ & $\begin{array}{c}1.22 \\
\pm 0.05\end{array}$ & $\begin{array}{c}0.23 \\
\pm 0.005 \\
\end{array}$ & $\begin{array}{c}0.20 \\
\pm 0.01 \\
\end{array}$ & - & $\begin{array}{c}0.07 \\
\pm 0.01 \\
\end{array}$ & $\begin{array}{c}0.14 \\
\pm 0.04\end{array}$ & - & - & - & - \\
\hline 3 & $\begin{array}{l}45.59 \\
\pm 0.19\end{array}$ & $\begin{array}{l}28.85 \\
\pm 0.18\end{array}$ & $\begin{array}{l}19.57 \\
\pm 0.24\end{array}$ & $\begin{array}{c}1.32 \\
\pm 0.03\end{array}$ & $\begin{array}{c}2.63 \\
\pm 0.13\end{array}$ & $\begin{array}{c}0.59 \\
\pm 0.03\end{array}$ & $\begin{array}{c}0.02 \\
\pm 0.006\end{array}$ & $\begin{array}{c}0.62 \\
\pm 0.04\end{array}$ & $\begin{array}{c}0.36 \\
\pm 0.006\end{array}$ & $\begin{array}{c}0.18 \\
\pm 0.01\end{array}$ & - & $\begin{array}{c}0.09 \\
\pm 0.02\end{array}$ & $\begin{array}{c}0.13 \\
\pm 0.04\end{array}$ & $\begin{array}{c}0.05 \\
\pm 0.007\end{array}$ & - & - & - \\
\hline 4 & $\begin{array}{l}50.50 \\
\pm 0.17\end{array}$ & $\begin{array}{l}23.89 \\
\pm 0.14\end{array}$ & $\begin{array}{l}19.35 \\
\pm 0.2\end{array}$ & $\begin{array}{c}2.56 \\
\pm 0.04\end{array}$ & $\begin{array}{c}2.31 \\
\pm 0.11\end{array}$ & $\begin{array}{c}0.55 \\
\pm 0.02\end{array}$ & $\begin{array}{c}0.07 \\
\pm 0.006 \\
\end{array}$ & $\begin{array}{c}0.38 \\
\pm 0.03\end{array}$ & $\begin{array}{c}0.23 \\
\pm 0.004 \\
\end{array}$ & $\begin{array}{c}0.13 \\
\pm 0.008 \\
\end{array}$ & - & - & - & $\begin{array}{c}0.03 \\
\pm 0.005 \\
\end{array}$ & $\begin{array}{c}0.07 \\
\pm 0.002 \\
\end{array}$ & - & - \\
\hline 5 & $\begin{array}{l}45.92 \\
\pm 0.2 \\
\end{array}$ & $\begin{array}{c}26.41 \\
\pm 0.17 \\
\end{array}$ & $\begin{array}{c}15.53 \\
\pm 0.25 \\
\end{array}$ & $\begin{array}{c}1.04 \\
\pm 0.03 \\
\end{array}$ & $\begin{array}{c}2.51 \\
\pm 0.14 \\
\end{array}$ & $\begin{array}{c}1.52 \\
\pm 0.03 \\
\end{array}$ & $\begin{array}{c}0.05 \\
\pm 0.007 \\
\end{array}$ & $\begin{array}{c}0.44 \\
\pm 0.04 \\
\end{array}$ & $\begin{array}{c}0.35 \\
\pm 0.007 \\
\end{array}$ & $\begin{array}{c}6.19 \\
\pm 0.07 \\
\end{array}$ & - & $\begin{array}{c}0.05 \\
\pm 0.02 \\
\end{array}$ & - & - & - & - & - \\
\hline 7 & $\begin{array}{l}27.90 \\
\pm 0.22 \\
\end{array}$ & $\begin{array}{l}44.72 \\
\pm 0.31 \\
\end{array}$ & $\begin{array}{c}17.16 \\
\pm 0.39 \\
\end{array}$ & $\begin{array}{c}0.93 \\
\pm 0.04 \\
\end{array}$ & $\begin{array}{c}1.94 \\
\pm 0.17 \\
\end{array}$ & $\begin{array}{c}1.23 \\
\pm 0.05 \\
\end{array}$ & $\begin{array}{c}0.13 \\
\pm 0.02 \\
\end{array}$ & $\begin{array}{c}0.48 \\
\pm 0.06 \\
\end{array}$ & $\begin{array}{c}0.90 \\
\pm 0.02 \\
\end{array}$ & $\begin{array}{c}4.26 \\
\pm 0.09 \\
\end{array}$ & - & - & $\begin{array}{c}0.35 \\
\pm 0.06 \\
\end{array}$ & - & $\begin{array}{c}0.08 \\
\pm 0.01 \\
\end{array}$ & - & - \\
\hline 8 & $\begin{array}{c}51.62 \\
\pm 0.17 \\
\end{array}$ & $\begin{array}{c}22.05 \\
\pm 0.14 \\
\end{array}$ & $\begin{array}{l}20.94 \\
\pm 0.19 \\
\end{array}$ & $\begin{array}{c}1.44 \\
\pm 0.03 \\
\end{array}$ & $\begin{array}{c}2.24 \\
\pm 0.11\end{array}$ & $\begin{array}{c}0.93 \\
\pm 0.02 \\
\end{array}$ & $\begin{array}{c}0.03 \\
\pm 0.004 \\
\end{array}$ & $\begin{array}{c}0.25 \\
\pm 0.03 \\
\end{array}$ & $\begin{array}{c}0.17 \\
\pm 0.004 \\
\end{array}$ & $\begin{array}{c}0.14 \\
\pm 0.009 \\
\end{array}$ & $\begin{array}{c}0.04 \\
\pm 0.008 \\
\end{array}$ & $\begin{array}{c}0.04 \\
\pm 0.01\end{array}$ & $\begin{array}{c}0.12 \\
\pm 0.03\end{array}$ & - & - & - & - \\
\hline 9 & $\begin{array}{c}32.50 \\
\pm 0.22 \\
\end{array}$ & $\begin{array}{l}48.55 \\
\pm 0.3 \\
\end{array}$ & $\begin{array}{c}11.08 \\
\pm 0.37 \\
\end{array}$ & $\begin{array}{c}0.88 \\
\pm 0.04 \\
\end{array}$ & $\begin{array}{c}3.24 \\
\pm 0.17 \\
\end{array}$ & $\begin{array}{c}1.56 \\
\pm 0.04 \\
\end{array}$ & $\begin{array}{c}0.06 \\
\pm 0.01 \\
\end{array}$ & $\begin{array}{c}0.47 \\
\pm 0.05 \\
\end{array}$ & $\begin{array}{c}0.86 \\
\pm 0.02 \\
\end{array}$ & $\begin{array}{c}0.49 \\
\pm 0.03 \\
\end{array}$ & - & - & $\begin{array}{c}0.20 \\
\pm 0.05 \\
\end{array}$ & $\begin{array}{c}0.11 \\
\pm 0.02 \\
\end{array}$ & - & $\begin{array}{c}0.16 \\
\pm 0.04 \\
\end{array}$ & - \\
\hline 10 & $\begin{array}{c}36.17 \\
\pm 0.15\end{array}$ & $\begin{array}{l}13.88 \\
\pm 0.1\end{array}$ & $\begin{array}{c}20.29 \\
\pm 0.24\end{array}$ & $\begin{array}{c}0.98 \\
\pm 0.02\end{array}$ & $\begin{array}{c}1.50 \\
\pm 0.08\end{array}$ & $\begin{array}{c}0.92 \\
\pm 0.02\end{array}$ & $\begin{array}{c}0.02 \\
\pm 0.005 \\
\end{array}$ & $\begin{array}{c}0.15 \\
\pm 0.02\end{array}$ & $\begin{array}{c}0.24 \\
\pm 0.005 \\
\end{array}$ & $\begin{array}{c}25.72 \\
\pm 0.12 \\
\end{array}$ & - & $\begin{array}{c}0.02 \\
\pm 0.007 \\
\end{array}$ & $\begin{array}{c}0.11 \\
\pm 0.02\end{array}$ & - & - & - & - \\
\hline 11 & $\begin{array}{l}51.96 \\
\pm 0.17 \\
\end{array}$ & $\begin{array}{c}20.09 \\
\pm 0.13 \\
\end{array}$ & $\begin{array}{c}21.59 \\
\pm 0.18 \\
\end{array}$ & $\begin{array}{c}1.40 \\
\pm 0.03 \\
\end{array}$ & $\begin{array}{c}2.37 \\
\pm 0.12 \\
\end{array}$ & $\begin{array}{c}1.51 \\
\pm 0.03 \\
\end{array}$ & $\begin{array}{c}0.35 \\
\pm 0.01 \\
\end{array}$ & $\begin{array}{c}0.28 \\
\pm 0.03 \\
\end{array}$ & $\begin{array}{c}0.18 \\
\pm 0.004 \\
\end{array}$ & $\begin{array}{c}0.12 \\
\pm 0.008 \\
\end{array}$ & - & - & $\begin{array}{c}0.15 \\
\pm 0.04 \\
\end{array}$ & - & - & - & - \\
\hline 12 & $\begin{array}{l}45.95 \\
\pm 0.17\end{array}$ & $\begin{array}{c}24.74 \\
\pm 0.16\end{array}$ & $\begin{array}{c}18.16 \\
\pm 0.21\end{array}$ & $\begin{array}{c}3.10 \\
\pm 0.04\end{array}$ & $\begin{array}{c}2.17 \\
\pm 0.12\end{array}$ & $\begin{array}{c}4.76 \\
\pm 0.04\end{array}$ & $\begin{array}{c}0.05 \\
\pm 0.006\end{array}$ & $\begin{array}{c}0.49 \\
\pm 0.04\end{array}$ & $\begin{array}{c}0.24 \\
\pm 0.005\end{array}$ & $\begin{array}{c}0.15 \\
\pm 0.01\end{array}$ & - & - & $\begin{array}{c}0.15 \\
\pm 0.04\end{array}$ & $\begin{array}{c}0.04 \\
\pm 0.006\end{array}$ & - & - & - \\
\hline
\end{tabular}


Table 2. Cont.

\begin{tabular}{|c|c|c|c|c|c|c|c|c|c|c|c|c|c|c|c|c|c|}
\hline Object Number & $\mathrm{Si}$ & $\mathrm{Fe}$ & Al & $\mathbf{P}$ & $\mathrm{Ti}$ & $S$ & $\mathbf{P b}$ & Mn & $\mathrm{Zr}$ & $\mathrm{Zn}$ & $\mathrm{Cu}$ & $\mathrm{Ni}$ & $\mathbf{V}$ & $\mathbf{B i}$ & $\mathrm{Nb}$ & $\mathrm{Cr}$ & Sn \\
\hline 13 & $\begin{array}{l}53.98 \\
\pm 0.15\end{array}$ & $\begin{array}{l}17.26 \\
\pm 0.1\end{array}$ & $\begin{array}{l}22.51 \\
\pm 0.17\end{array}$ & $\begin{array}{c}2.01 \\
\pm 0.03\end{array}$ & $\begin{array}{c}1.66 \\
\pm 0.08\end{array}$ & $\begin{array}{c}1.82 \\
\pm 0.03\end{array}$ & $\begin{array}{c}0.05 \\
\pm 0.004\end{array}$ & $\begin{array}{c}0.34 \\
\pm 0.02\end{array}$ & $\begin{array}{c}0.14 \\
\pm 0.003\end{array}$ & $\begin{array}{c}0.08 \\
\pm 0.005\end{array}$ & $\begin{array}{c}0.02 \\
\pm 0.005\end{array}$ & $\begin{array}{c}0.03 \\
\pm 0.007\end{array}$ & $\begin{array}{c}0.10 \\
\pm 0.03\end{array}$ & - & - & - & - \\
\hline 14 & $\begin{array}{l}49.84 \\
\pm 0.16\end{array}$ & $\begin{array}{l}22.05 \\
\pm 0.13\end{array}$ & $\begin{array}{l}23.11 \\
\pm 0.19\end{array}$ & $\begin{array}{c}1.23 \\
\pm 0.03\end{array}$ & $\begin{array}{l}2.21 \\
\pm 0.1\end{array}$ & $\begin{array}{c}0.78 \\
\pm 0.02\end{array}$ & $\begin{array}{c}0.03 \\
\pm 0.004\end{array}$ & $\begin{array}{c}0.46 \\
\pm 0.03\end{array}$ & $\begin{array}{c}0.16 \\
\pm 0.003\end{array}$ & $\begin{array}{c}0.08 \\
\pm 0.006\end{array}$ & - & $\begin{array}{c}0.05 \\
\pm 0.01\end{array}$ & - & - & - & - & - \\
\hline 15 & $\begin{array}{l}55.29 \\
\pm 0.16\end{array}$ & $\begin{array}{l}16.91 \\
\pm 0.11\end{array}$ & $\begin{array}{l}22.36 \\
\pm 0.16\end{array}$ & $\begin{array}{c}2.14 \\
\pm 0.03\end{array}$ & $\begin{array}{l}2.23 \\
\pm 0.1\end{array}$ & $\begin{array}{c}0.41 \\
\pm 0.02\end{array}$ & $\begin{array}{c}0.02 \\
\pm 0.003\end{array}$ & $\begin{array}{c}0.24 \\
\pm 0.02\end{array}$ & $\begin{array}{c}0.16 \\
\pm 0.003\end{array}$ & $\begin{array}{c}0.09 \\
\pm 0.006\end{array}$ & - & $\begin{array}{c}0.03 \\
\pm 0.008\end{array}$ & $\begin{array}{c}0.13 \\
\pm 0.03\end{array}$ & - & - & - & - \\
\hline 16 & $\begin{array}{l}43.63 \\
\pm 0.18\end{array}$ & $\begin{array}{l}30.69 \\
\pm 0.18\end{array}$ & $\begin{array}{l}19.67 \\
\pm 0.22\end{array}$ & $\begin{array}{c}1.70 \\
\pm 0.03\end{array}$ & $\begin{array}{c}2.65 \\
\pm 0.14\end{array}$ & $\begin{array}{c}0.74 \\
\pm 0.03\end{array}$ & $\begin{array}{c}0.03 \\
\pm 0.006\end{array}$ & $\begin{array}{c}0.34 \\
\pm 0.03\end{array}$ & $\begin{array}{c}0.29 \\
\pm 0.006\end{array}$ & $\begin{array}{c}0.14 \\
\pm 0.01\end{array}$ & - & - & $\begin{array}{c}0.12 \\
\pm 0.04\end{array}$ & - & $\begin{array}{c}0.03 \\
\pm 0.003\end{array}$ & - & - \\
\hline 17 & $\begin{array}{l}49.63 \\
\pm 0.15\end{array}$ & $\begin{array}{l}18.44 \\
\pm 0.12\end{array}$ & $\begin{array}{l}22.54 \\
\pm 0.17\end{array}$ & $\begin{array}{c}5.90 \\
\pm 0.05\end{array}$ & $\begin{array}{l}1.88 \\
\pm 0.1 \\
\end{array}$ & $\begin{array}{c}0.65 \\
\pm 0.02\end{array}$ & $\begin{array}{c}0.03 \\
\pm 0.003\end{array}$ & $\begin{array}{c}0.57 \\
\pm 0.03\end{array}$ & $\begin{array}{c}0.17 \\
\pm 0.003\end{array}$ & $\begin{array}{c}0.06 \\
\pm 0.005\end{array}$ & - & - & $\begin{array}{c}0.11 \\
\pm 0.03\end{array}$ & $\begin{array}{c}0.03 \\
\pm 0.004 \\
\end{array}$ & $\begin{array}{c}0.01 \\
\pm 0.002 \\
\end{array}$ & - & - \\
\hline 18 & $\begin{array}{l}49.51 \\
\pm 0.16\end{array}$ & $\begin{array}{l}19.84 \\
\pm 0.12\end{array}$ & $\begin{array}{l}25.12 \\
\pm 0.18\end{array}$ & $\begin{array}{c}1.51 \\
\pm 0.03\end{array}$ & $\begin{array}{l}2.10 \\
\pm 0.1\end{array}$ & $\begin{array}{c}1.24 \\
\pm 0.03\end{array}$ & $\begin{array}{c}0.01 \\
\pm 0.003\end{array}$ & $\begin{array}{c}0.30 \\
\pm 0.02\end{array}$ & - & $\begin{array}{c}0.09 \\
\pm 0.007\end{array}$ & - & $\begin{array}{c}0.04 \\
\pm 0.009\end{array}$ & $\begin{array}{c}0.21 \\
\pm 0.03\end{array}$ & $\begin{array}{c}0.03 \\
\pm 0.004\end{array}$ & $\begin{array}{c}0.01 \\
\pm 0.002\end{array}$ & - & - \\
\hline 19 & $\begin{array}{l}56.56 \\
\pm 0.16\end{array}$ & $\begin{array}{l}15.47 \\
\pm 0.11\end{array}$ & $\begin{array}{l}22.98 \\
\pm 0.16\end{array}$ & $\begin{array}{c}1.49 \\
\pm 0.03\end{array}$ & $\begin{array}{l}2.08 \\
\pm 0.1\end{array}$ & $\begin{array}{c}0.75 \\
\pm 0.02\end{array}$ & $\begin{array}{c}0.02 \\
\pm 0.003\end{array}$ & $\begin{array}{c}0.22 \\
\pm 0.02\end{array}$ & $\begin{array}{c}0.14 \\
\pm 0.003\end{array}$ & $\begin{array}{c}0.11 \\
\pm 0.006\end{array}$ & $\begin{array}{c}0.03 \\
\pm 0.005\end{array}$ & - & $\begin{array}{c}0.13 \\
\pm 0.03\end{array}$ & $\begin{array}{c}0.02 \\
\pm 0.003\end{array}$ & - & - & - \\
\hline 20 & $\begin{array}{l}47.09 \\
\pm 0.15 \\
\end{array}$ & $\begin{array}{l}20.37 \\
\pm 0.12 \\
\end{array}$ & $\begin{array}{l}20.52 \\
\pm 0.18\end{array}$ & $\begin{array}{c}4.82 \\
\pm 0.04\end{array}$ & $\begin{array}{l}2.04 \\
\pm 0.1 \\
\end{array}$ & $\begin{array}{c}4.26 \\
\pm 0.04 \\
\end{array}$ & $\begin{array}{c}0.02 \\
\pm 0.003 \\
\end{array}$ & $\begin{array}{c}0.36 \\
\pm 0.02 \\
\end{array}$ & $\begin{array}{c}0.16 \\
\pm 0.003 \\
\end{array}$ & $\begin{array}{c}0.11 \\
\pm 0.007 \\
\end{array}$ & - & $\begin{array}{c}0.04 \\
\pm 0.008 \\
\end{array}$ & $\begin{array}{c}0.19 \\
\pm 0.03 \\
\end{array}$ & $\begin{array}{c}0.03 \\
\pm 0.003 \\
\end{array}$ & $\begin{array}{c}0.01 \\
\pm 0.002 \\
\end{array}$ & - & - \\
\hline 21 & $\begin{array}{l}49.82 \\
\pm 0.19\end{array}$ & $\begin{array}{l}24.84 \\
\pm 0.15\end{array}$ & $\begin{array}{l}19.44 \\
\pm 0.21\end{array}$ & $\begin{array}{c}1.57 \\
\pm 0.03\end{array}$ & $\begin{array}{c}2.65 \\
\pm 0.13\end{array}$ & $\begin{array}{c}0.6 \\
\pm 0.02\end{array}$ & $\begin{array}{c}0.03 \\
\pm 0.005\end{array}$ & $\begin{array}{c}0.41 \\
\pm 0.03\end{array}$ & $\begin{array}{c}0.21 \\
\pm 0.004\end{array}$ & $\begin{array}{c}0.16 \\
\pm 0.01\end{array}$ & - & $\begin{array}{c}0.05 \\
\pm 0.01\end{array}$ & $\begin{array}{c}0.19 \\
\pm 0.04\end{array}$ & $\begin{array}{c}0.04 \\
\pm 0.005\end{array}$ & - & - & - \\
\hline 22 & $\begin{array}{l}49.08 \\
\pm 0.16\end{array}$ & $\begin{array}{l}20.06 \\
\pm 0.12\end{array}$ & $\begin{array}{l}22.92 \\
\pm 0.18\end{array}$ & $\begin{array}{c}3.38 \\
\pm 0.04\end{array}$ & $\begin{array}{l}2.16 \\
\pm 0.1\end{array}$ & $\begin{array}{c}1.69 \\
\pm 0.03\end{array}$ & $\begin{array}{c}0.04 \\
\pm 0.004\end{array}$ & $\begin{array}{c}0.29 \\
\pm 0.02\end{array}$ & $\begin{array}{c}0.13 \\
\pm 0.003\end{array}$ & $\begin{array}{c}0.10 \\
\pm 0.006\end{array}$ & - & $\begin{array}{c}0.03 \\
\pm 0.008\end{array}$ & $\begin{array}{c}0.12 \\
\pm 0.03\end{array}$ & - & - & - & - \\
\hline 23 & $\begin{array}{l}55.16 \\
\pm 0.15\end{array}$ & $\begin{array}{l}16.75 \\
\pm 0.11\end{array}$ & $\begin{array}{l}24.04 \\
\pm 0.16\end{array}$ & $\begin{array}{c}1.09 \\
\pm 0.02\end{array}$ & $\begin{array}{l}2.08 \\
\pm 0.1 \\
\end{array}$ & $\begin{array}{c}0.38 \\
\pm 0.02\end{array}$ & $\begin{array}{c}0.01 \\
\pm 0.003\end{array}$ & $\begin{array}{c}0.24 \\
\pm 0.02\end{array}$ & $\begin{array}{c}0.14 \\
\pm 0.003\end{array}$ & $\begin{array}{c}0.05 \\
\pm 0.005\end{array}$ & - & $\begin{array}{c}0.02 \\
\pm 0.007\end{array}$ & - & $\begin{array}{c}0.03 \\
\pm 0.003\end{array}$ & $\begin{array}{c}0.01 \\
\pm 0.002\end{array}$ & - & - \\
\hline 24 & $\begin{array}{l}55.09 \\
\pm 0.16\end{array}$ & $\begin{array}{l}17.22 \\
\pm 0.11\end{array}$ & $\begin{array}{l}22.51 \\
\pm 0.16\end{array}$ & $\begin{array}{c}1.99 \\
\pm 0.03\end{array}$ & $\begin{array}{l}2.20 \\
\pm 0.1\end{array}$ & $\begin{array}{c}0.38 \\
\pm 0.02\end{array}$ & $\begin{array}{c}0.03 \\
\pm 0.003\end{array}$ & $\begin{array}{c}0.23 \\
\pm 0.02\end{array}$ & $\begin{array}{c}0.18 \\
\pm 0.003\end{array}$ & $\begin{array}{c}0.05 \\
\pm 0.005\end{array}$ & - & $\begin{array}{c}0.04 \\
\pm 0.008\end{array}$ & $\begin{array}{c}0.09 \\
\pm 0.03\end{array}$ & - & $\begin{array}{c}0.01 \\
\pm 0.002\end{array}$ & $\begin{array}{c}0.08 \\
\pm 0.02\end{array}$ & - \\
\hline 25 & $\begin{array}{l}48.25 \\
\pm 0.15\end{array}$ & $\begin{array}{l}19.75 \\
\pm 0.12\end{array}$ & $\begin{array}{l}21.52 \\
\pm 0.18\end{array}$ & $\begin{array}{c}3.64 \\
\pm 0.04\end{array}$ & $\begin{array}{l}2.26 \\
\pm 0.1 \\
\end{array}$ & $\begin{array}{c}3.70 \\
\pm 0.04\end{array}$ & $\begin{array}{c}0.03 \\
\pm 0.004\end{array}$ & $\begin{array}{c}0.41 \\
\pm 0.03\end{array}$ & $\begin{array}{c}0.18 \\
\pm 0.003\end{array}$ & $\begin{array}{c}0.13 \\
\pm 0.007\end{array}$ & - & $\begin{array}{c}0.03 \\
\pm 0.008\end{array}$ & $\begin{array}{c}0.11 \\
\pm 0.03\end{array}$ & - & $\begin{array}{c}0.01 \\
\pm 0.002 \\
\end{array}$ & - & - \\
\hline 26 & $\begin{array}{l}46.77 \\
\pm 0.18 \\
\end{array}$ & $\begin{array}{l}26.33 \\
\pm 0.15 \\
\end{array}$ & $\begin{array}{l}18.17 \\
\pm 0.22 \\
\end{array}$ & $\begin{array}{c}2.88 \\
\pm 0.04 \\
\end{array}$ & $\begin{array}{c}2.82 \\
\pm 0.12 \\
\end{array}$ & $\begin{array}{c}1.83 \\
\pm 0.03 \\
\end{array}$ & $\begin{array}{c}0.01 \\
\pm 0.004 \\
\end{array}$ & $\begin{array}{c}0.61 \\
\pm 0.03 \\
\end{array}$ & $\begin{array}{c}0.18 \\
\pm 0.004 \\
\end{array}$ & $\begin{array}{c}0.34 \\
\pm 0.01 \\
\end{array}$ & - & $\begin{array}{c}0.06 \\
\pm 0.01 \\
\end{array}$ & - & - & - & - & - \\
\hline 27 & $\begin{array}{l}49.40 \\
\pm 0.17\end{array}$ & $\begin{array}{l}20.07 \\
\pm 0.13\end{array}$ & $\begin{array}{l}23.56 \\
\pm 0.19\end{array}$ & $\begin{array}{c}2.25 \\
\pm 0.03\end{array}$ & $\begin{array}{c}2.23 \\
\pm 0.11\end{array}$ & $\begin{array}{c}1.50 \\
\pm 0.03\end{array}$ & $\begin{array}{c}0.01 \\
\pm 0.003\end{array}$ & $\begin{array}{c}0.5 \\
\pm 0.03\end{array}$ & $\begin{array}{c}0.16 \\
\pm 0.003\end{array}$ & $\begin{array}{c}0.10 \\
\pm 0.007\end{array}$ & - & $\begin{array}{c}0.04 \\
\pm 0.01\end{array}$ & $\begin{array}{c}0.16 \\
\pm 0.03\end{array}$ & $\begin{array}{c}0.03 \\
\pm 0.004\end{array}$ & - & - & - \\
\hline 28 & $\begin{array}{l}45.94 \\
\pm 0.18\end{array}$ & $\begin{array}{l}28.24 \\
\pm 0.16\end{array}$ & $\begin{array}{l}18.97 \\
\pm 0.22\end{array}$ & $\begin{array}{c}2.08 \\
\pm 0.03\end{array}$ & $\begin{array}{c}2.22 \\
\pm 0.12\end{array}$ & $\begin{array}{c}1.13 \\
\pm 0.03\end{array}$ & $\begin{array}{c}0.05 \\
\pm 0.006\end{array}$ & $\begin{array}{c}0.61 \\
\pm 0.04\end{array}$ & $\begin{array}{c}0.24 \\
\pm 0.005\end{array}$ & $\begin{array}{c}0.16 \\
\pm 0.01\end{array}$ & $\begin{array}{c}0.07 \\
\pm 0.01\end{array}$ & $\begin{array}{c}0.11 \\
\pm 0.02\end{array}$ & $\begin{array}{c}0.18 \\
\pm 0.04\end{array}$ & - & - & - & - \\
\hline
\end{tabular}


Table 2. Cont.

\begin{tabular}{|c|c|c|c|c|c|c|c|c|c|c|c|c|c|c|c|c|c|}
\hline Object Number & $\mathrm{Si}$ & $\mathrm{Fe}$ & Al & $\mathbf{P}$ & $\mathrm{Ti}$ & $\mathrm{S}$ & $\mathrm{Pb}$ & Mn & $\mathrm{Zr}$ & $\mathrm{Zn}$ & $\mathrm{Cu}$ & $\mathrm{Ni}$ & $\mathrm{V}$ & $\mathbf{B i}$ & $\mathrm{Nb}$ & $\mathrm{Cr}$ & $\mathrm{Sn}$ \\
\hline 29 & $\begin{array}{l}47.10 \\
\pm 0.18\end{array}$ & $\begin{array}{l}26.51 \\
\pm 0.16\end{array}$ & $\begin{array}{l}20.15 \\
\pm 0.22\end{array}$ & $\begin{array}{c}1.28 \\
\pm 0.03\end{array}$ & $\begin{array}{c}2.15 \\
\pm 0.12\end{array}$ & $\begin{array}{c}1.94 \\
\pm 0.03\end{array}$ & $\begin{array}{c}0.06 \\
\pm 0.006\end{array}$ & $\begin{array}{c}0.24 \\
\pm 0.03\end{array}$ & $\begin{array}{c}0.25 \\
\pm 0.005\end{array}$ & $\begin{array}{c}0.14 \\
\pm 0.01\end{array}$ & - & $\begin{array}{c}0.07 \\
\pm 0.02\end{array}$ & $\begin{array}{c}0.11 \\
\pm 0.04\end{array}$ & - & - & - & - \\
\hline 30 & $\begin{array}{c}47.55 \\
\pm 0.19\end{array}$ & $\begin{array}{c}27.97 \\
\pm 0.16\end{array}$ & $\begin{array}{c}18.61 \\
\pm 0.23\end{array}$ & $\begin{array}{c}1.39 \\
\pm 0.03\end{array}$ & $\begin{array}{c}2.70 \\
\pm 0.13\end{array}$ & $\begin{array}{c}0.75 \\
\pm 0.03\end{array}$ & $\begin{array}{c}0.03 \\
\pm 0.005\end{array}$ & $\begin{array}{c}0.33 \\
\pm 0.03\end{array}$ & $\begin{array}{c}0.26 \\
\pm 0.005\end{array}$ & $\begin{array}{c}0.13 \\
\pm 0.01\end{array}$ & - & $\begin{array}{c}0.06 \\
\pm 0.01\end{array}$ & $\begin{array}{c}0.18 \\
\pm 0.04\end{array}$ & $\begin{array}{c}0.04 \\
\pm 0.006\end{array}$ & $\begin{array}{c}0.02 \\
\pm 0.003\end{array}$ & - & - \\
\hline 31 & $\begin{array}{c}61.39 \\
\pm 0.17\end{array}$ & $\begin{array}{l}9.94 \\
\pm 0.1\end{array}$ & $\begin{array}{c}20.95 \\
\pm 0.16\end{array}$ & $\begin{array}{c}2.40 \\
\pm 0.04\end{array}$ & $\begin{array}{c}2.29 \\
\pm 0.12\end{array}$ & $\begin{array}{c}2.28 \\
\pm 0.03\end{array}$ & $\begin{array}{c}0.03 \\
\pm 0.003\end{array}$ & $\begin{array}{c}0.33 \\
\pm 0.03\end{array}$ & $\begin{array}{c}0.17 \\
\pm 0.003\end{array}$ & $\begin{array}{c}0.07 \\
\pm 0.006\end{array}$ & - & - & $\begin{array}{c}0.15 \\
\pm 0.04\end{array}$ & - & - & - & - \\
\hline 32 & $\begin{array}{c}50.49 \\
\pm 0.18\end{array}$ & $\begin{array}{c}23.5 \\
\pm 0.14\end{array}$ & $\begin{array}{l}21.22 \\
\pm 0.2\end{array}$ & $\begin{array}{c}0.86 \\
\pm 0.02\end{array}$ & $\begin{array}{c}1.92 \\
\pm 0.11\end{array}$ & $\begin{array}{c}0.36 \\
\pm 0.02\end{array}$ & $\begin{array}{c}0.04 \\
\pm 0.005\end{array}$ & $\begin{array}{c}1.17 \\
\pm 0.05\end{array}$ & $\begin{array}{c}0.20 \\
\pm 0.004\end{array}$ & $\begin{array}{c}0.13 \\
\pm 0.009\end{array}$ & - & $\begin{array}{c}0.11 \\
\pm 0.01\end{array}$ & - & - & - & - & - \\
\hline 33 & $\begin{array}{c}48.93 \\
\pm 0.15\end{array}$ & $\begin{array}{c}20.65 \\
\pm 0.12\end{array}$ & $\begin{array}{l}21.66 \\
\pm 0.18\end{array}$ & $\begin{array}{c}2.96 \\
\pm 0.03\end{array}$ & $\begin{array}{l}2.05 \\
\pm 0.1\end{array}$ & $\begin{array}{c}2.90 \\
\pm 0.03\end{array}$ & $\begin{array}{c}0.04 \\
\pm 0.004\end{array}$ & $\begin{array}{c}0.31 \\
\pm 0.02\end{array}$ & $\begin{array}{c}0.19 \\
\pm 0.003\end{array}$ & $\begin{array}{c}0.11 \\
\pm 0.007\end{array}$ & $\begin{array}{c}0.03 \\
\pm 0.006\end{array}$ & $\begin{array}{c}0.04 \\
\pm 0.009\end{array}$ & $\begin{array}{c}0.14 \\
\pm 0.03\end{array}$ & - & $\begin{array}{c}0.01 \\
\pm 0.002\end{array}$ & - & - \\
\hline 34 & $\begin{array}{l}48.08 \\
\pm 0.17\end{array}$ & $\begin{array}{c}21.36 \\
\pm 0.14\end{array}$ & $\begin{array}{l}18.75 \\
\pm 0.2\end{array}$ & $\begin{array}{c}6.96 \\
\pm 0.05\end{array}$ & $\begin{array}{c}2.07 \\
\pm 0.12\end{array}$ & $\begin{array}{c}1.86 \\
\pm 0.03\end{array}$ & $\begin{array}{c}0.07 \\
\pm 0.006\end{array}$ & $\begin{array}{c}0.4 \\
\pm 0.03\end{array}$ & $\begin{array}{c}0.19 \\
\pm 0.004\end{array}$ & $\begin{array}{c}0.13 \\
\pm 0.009\end{array}$ & - & - & $\begin{array}{c}0.14 \\
\pm 0.04\end{array}$ & - & $\begin{array}{c}0.02 \\
\pm 0.002\end{array}$ & - & - \\
\hline
\end{tabular}


According to the data obtained from the surfaces, there are 17 chemical elements that have been determined using a semi-quantitative express analysis with a handheld pXRF spectrometer. Different concentrations of chemical elements indicate different intensities regarding an element's presence on the mold's surfaces.

The main elements of the clay-ceramic composition $(\mathrm{Si}, \mathrm{Fe}, \mathrm{Al}$, and $\mathrm{Ti}$ ) show a consistently high concentration on both the working and non-working surfaces of the molds, on objects both with and without evidence of carbonization.

The presence of $\mathrm{Pb}$ traces in concentrations from 1 up to $15.1 \%$ on the working surfaces was observed among the metal elements assumed to be from the alloy composition (Figure 7a). The dispersion of the concentration values in the box-and-whisker plots (Figure 7c) indicates that $50 \%$ of the concentration of $\mathrm{Pb}$ lies within the interquartile range. Most of the values lie below the median line. The values with concentrations above $3.5 \%$ strongly indicate a good preservation of $\mathrm{Pb}$ residues in the surface layer of the working side.

According to the data obtained from the non-working side of the casting mold, it contains $\mathrm{Pb}$ in a much lower concentration (Table 2; Figure $7 \mathrm{~b}, \mathrm{~d}$ ). Most of the Pb concentrations obtained by screening the non-working surface are in the range up to $0.2 \%$, which indicates the $\mathrm{Pb}$ content of the ceramic clay material.

$\mathrm{Cu}$ is characteristically present on the working sides of four objects (number 8, 13, 28, and 33) at tenths of a percent and on the non-working sides at hundredths of a percent. As with lead, copper is characterized by differences in the concentrations on the working and non-working sides (Table 2; Figure 7a,b).

The presence of Sn was determined for only two objects (number 13 and 34) on the working side (Table 2; Figure 7a).

According to the data (Table 2) obtained by a screening pXRF analysis of the working (Figure 7a) and non-working (Figure $7 \mathrm{~b}$ ) sides of the molds, most of the concentration values for $\mathrm{Zn}$ lie in a similar range (Figure $7 \mathrm{c}, \mathrm{d}$ ). The exceptions are the values obtained from the surface of the object numbers 5, 6,7, and 10, which have residues of the medical bandage used as tags. Medical bandages often contain zinc oxide, which is the reason for its presence on the surface. The good preservation of this layer is due to the good preservation of the tissue backing with the adhesive layer. Thus, a comparison of the $\mathrm{Zn}$ intensities on the working and non-working sides (as shown for the case of $\mathrm{Pb}$ residues) does not allow us to consider $\mathrm{Zn}$ as a probable component of the copper-alloy cast in the molds.

The non-metals $\mathrm{P}$ and $\mathrm{S}$ show constant presence on the working and non-working sides. $\mathrm{P}$ has consistently higher concentrations on the working surfaces (up to 15\%) (Table 2).

The metals $\mathrm{Ni}, \mathrm{Bi}$, and $\mathrm{Nb}$ are found at the levels of hundredths of a percent (Table 2). $\mathrm{V}$ is established in the majority of objects, but only at the level of tenths of a percent. $\mathrm{Cr}$ is present in a small number of objects, in tenths and hundredths of a percent.

Additionally, $\mathrm{Mn}$ and $\mathrm{Zr}$ are registered on the surface of the forms in similar concentration ranges with a similar scatter pattern of values and refer to the composition of the ceramic material (Table 2).

\subsection{Results of SEM-EDS Examination of Ceramic Molds Surfaces}

SEM observation of the mold casting cavities showed differences in the ceramic substance microstructure of non-carbonized and carbonized surfaces. Non-carbonized ceramic surfaces do not demonstrate thermal transformation such as sintering, bubbling, and corrosion, as was noted above (Figure 6a,b). On the contrary, carbonized surfaces present features such as bubbling, corrosion, and sintering, caused certainly by high temperature impact on clay particles from ceramic substance during the casting process (Figure 6d,c).

The results of EDS examination are shown in Table 3, containing data on the range of weight values of different chemical elements in the objects examined. The data are grouped into two blocks. The upper block includes objects with carbonized working surfaces (numbers 13-34). The lower block concerns objects without evidence of carbonization (numbers 16-26). Additionally, Table 4 contains data on the selected EDS spectra compositions for objects with carbonized surfaces. 
Table 3. Results of the EDS examination of the sampled objects.

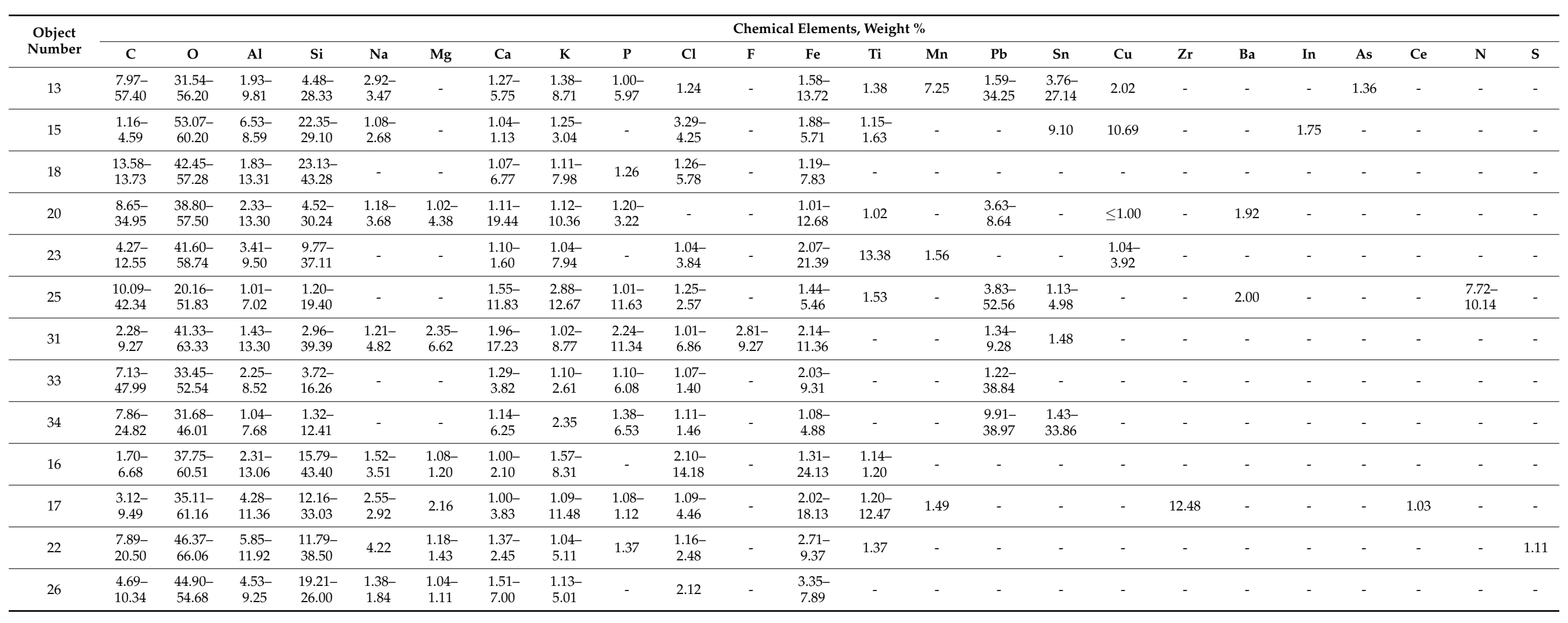


Table 4. Data on the selected EDS spectral compositions for objects with carbonized surfaces.

\begin{tabular}{|c|c|c|c|c|c|c|c|c|c|c|c|c|c|c|c|c|c|c|c|c|c|}
\hline \multirow{2}{*}{$\begin{array}{l}\text { Spectrum, } \\
\text { Number }\end{array}$} & \multicolumn{21}{|c|}{ Chemical Elements, Weight \% } \\
\hline & $\mathrm{C}$ & $\mathbf{O}$ & Al & $\mathrm{Si}$ & $\mathrm{Na}$ & $\mathrm{Mg}$ & $\mathrm{Ca}$ & K & $\mathbf{P}$ & $\mathrm{Cl}$ & $\mathrm{Fe}$ & $\mathrm{Ti}$ & Mn & $\mathrm{Pb}$ & Sn & $\mathrm{Cu}$ & Ba & In & As & $\mathrm{S}$ & $\mathbf{N}$ \\
\hline $\mathrm{N} 13 / 1 / 2$ & 9.07 & 44.12 & 4.94 & 11.49 & 0.42 & 0.19 & 1.05 & 1.62 & 0.18 & 0.25 & 2.24 & 0.22 & - & 15.05 & 1.91 & 0.32 & - & - & 1.36 & - & 5.60 \\
\hline $\mathrm{N} 13 / 1 / 3$ & 16.86 & 34.34 & 4.23 & 9.94 & 0.31 & 0.42 & 2.13 & 1.62 & 0.34 & 0.36 & 10.64 & 0.63 & - & 12.02 & 3.61 & 2.02 & - & - & 0.52 & - & - \\
\hline $\mathrm{N} 13 / 1 / 7$ & 17.72 & 46.26 & 4.00 & 10.37 & 0.31 & 0.32 & 1.42 & 1.20 & 0.35 & 0.20 & 3.20 & 0.30 & - & 6.43 & 1.87 & - & - & - & 0.59 & - & 5.46 \\
\hline $\mathrm{N} 13 / 4 / 13$ & 13.91 & 36.59 & 3.69 & 8.33 & 0.18 & 0.37 & 3.09 & 1.22 & 4.48 & 0.55 & 4.77 & 0.25 & - & 22.57 & - & - & - & - & - & - & - \\
\hline $\mathrm{N} 13 / 4 / 19$ & & 52.90 & 9.00 & 26.29 & 3.14 & 0.38 & 3.61 & 1.33 & 0.51 & 1.24 & 3.23 & 0.19 & - & 34.25 & - & - & - & - & - & - & - \\
\hline N15/1/1 & - & 60.20 & 6.90 & 22.35 & 0.51 & 0.44 & 1.04 & - & 0.33 & 3.29 & 2.93 & 0.25 & - & - & 9.10 & 10.69 & - & 1.75 & - & 0.23 & - \\
\hline $\mathrm{N} 15 / 2 / 6$ & - & 48.60 & 9.51 & 24.74 & 0.45 & 0.69 & 2.36 & 3.90 & 1.12 & 0.56 & 5.03 & 0.92 & - & - & - & 0.60 & - & - & - & - & - \\
\hline $\mathrm{N} 20 / 1 / 3$ & 9.73 & 50.71 & 8.29 & 17.92 & 0.64 & 0.98 & 1.44 & 0.90 & 0.41 & - & 7.90 & 0.86 & 0.12 & - & - & 0.20 & - & - & - & - & - \\
\hline $\mathrm{N} 20 / 1 / 8$ & 10.06 & 53.70 & 7.57 & 16.03 & 0.79 & 1.01 & 1.24 & 0.98 & 0.69 & - & 7.29 & 0.25 & 0.12 & - & - & 0.27 & - & - & - & - & - \\
\hline $\mathrm{N} 20 / 3 / 10$ & 21.14 & 38.80 & 5.72 & 17.16 & 0.28 & 0.21 & 1.43 & 7.17 & 1.91 & 0.17 & 1.47 & - & - & 3.63 & - & - & 0.92 & - & - & - & - \\
\hline $\mathrm{N} 20 / 3 / 9$ & 19.37 & 39.64 & 4.66 & 13.20 & 0.27 & 0.31 & 2.80 & 4.58 & 3.22 & 0.39 & 2.55 & 0.37 & - & 8.64 & - & - & - & - & - & - & - \\
\hline $\mathrm{N} 23 / 1 / 2$ & 7.99 & 45.79 & 7.40 & 24.20 & 0.44 & 0.20 & 0.61 & 7.94 & 0.22 & 1.04 & 3.38 & 0.28 & - & - & - & 0.51 & - & - & - & - & - \\
\hline $\mathrm{N} 23 / 1 / 5$ & - & 56.27 & 6.68 & 25.40 & 0.43 & 0.47 & 0.67 & 2.55 & 0.20 & 1.83 & 2.88 & 0.28 & - & - & - & 2.33 & - & - & - & - & - \\
\hline $\mathrm{N} 23 / 1 / 6$ & - & 48.51 & 7.77 & 26.58 & 0.39 & 0.48 & 1.45 & 3.30 & 0.26 & 2.04 & 8.06 & 0.62 & - & - & - & 0.55 & - & - & - & - & - \\
\hline $\mathrm{N} 25 / 1 / 4$ & - & 36.35 & 1.49 & 1.85 & - & 0.23 & 7.85 & - & 7.87 & 1.79 & 1.69 & - & - & 37.09 & 3.78 & - & - & - & - & - & - \\
\hline $\mathrm{N} 25 / 1 / 9$ & 19.11 & 36.43 & 1.71 & 3.19 & 0.23 & 0.28 & 5.88 & - & 5.39 & 1.14 & 1.96 & - & - & 23.18 & 2.93 & - & - & - & - & - & - \\
\hline $\mathrm{N} 25 / 2 / 1$ & 15.92 & 35.44 & 2.18 & 4.63 & - & 0.41 & 6.32 & - & 5.21 & 1.16 & 2.28 & - & - & 22.13 & 4.38 & - & - & - & - & - & - \\
\hline $\mathrm{N} 25 / 2 / 19$ & 20.19 & 39.42 & 2.51 & 6.54 & - & 0.33 & 6.75 & 0.61 & 5.51 & 0.96 & 3.12 & - & - & 19.95 & 3.48 & - & - & - & - & - & - \\
\hline N31/ 1/1 & - & 51.66 & 3.37 & 7.14 & 0.25 & 0.45 & 4.36 & 0.43 & 4.37 & 3.39 & 4.90 & 0.20 & - & 9.28 & 1.48 & - & - & - & - & - & 8.73 \\
\hline $\mathrm{N} 31 / 1 / 3$ & - & 56.41 & 3.94 & 6.62 & 0.39 & 0.98 & 3.51 & 0.23 & 4.08 & 3.51 & 3.70 & 0.17 & - & 6.57 & 0.91 & - & - & - & - & - & 8.11 \\
\hline $\mathrm{N} 31 / 1 / 14$ & - & 55.19 & 5.52 & 10.29 & 0.30 & 6.62 & 3.11 & 0.50 & 2.78 & 2.50 & 3.48 & 0.19 & - & 5.81 & 1.04 & - & - & - & - & - & - \\
\hline $\mathrm{N} 33 / 1 / 3$ & - & 39.41 & 2.76 & 4.39 & - & 0.24 & 3.57 & 0.41 & 6.08 & 1.40 & 2.30 & - & - & 38.84 & - & - & - & - & 0.60 & - & - \\
\hline $\mathrm{N} 33 / 1 / 8$ & - & 37.79 & 3.30 & 5.69 & - & 0.30 & 3.82 & 0.54 & 5.94 & 1.25 & 3.08 & - & - & 38.28 & - & - & - & - & - & - & - \\
\hline $\mathrm{N} 33 / 1 / 11$ & 9.31 & 48.31 & 5.56 & 11.30 & 0.25 & 0.53 & 2.03 & 1.10 & 2.75 & 0.40 & 4.36 & 0.33 & - & 13.77 & - & - & - & - & - & - & - \\
\hline $\mathrm{N} 34 / 1 / 3$ & 7.86 & 39.49 & 1.31 & 3.21 & - & - & 1.14 & - & 1.38 & - & 1.83 & - & - & 9.91 & 33.86 & - & - & - & - & - & - \\
\hline $\mathrm{N} 34 / 2 / 5$ & - & 46.01 & 2.81 & 5.52 & 0.37 & 0.30 & 4.04 & 0.59 & 6.07 & 1.46 & 2.15 & - & - & 37.97 & 3.91 & - & - & - & - & - & - \\
\hline $\mathrm{N} 34 / 2 / 6$ & 13.57 & 31.68 & 1.09 & 1.75 & 0.17 & 0.20 & 4.68 & - & 6.53 & 0.69 & 3.61 & - & - & 34.14 & 2.50 & - & - & - & - & - & - \\
\hline $\mathrm{N} 34 / 3 / 2$ & 20.11 & 36.34 & 2.41 & 4.64 & 0.26 & 0.25 & 3.43 & - & 2.95 & 0.30 & 2.69 & - & - & 12.39 & - & - & - & - & - & - & - \\
\hline $\mathrm{N} 34 / 3 / 6$ & 8.72 & 37.68 & 1.06 & 1.83 & 0.15 & - & 1.91 & - & 3.06 & 0.43 & 1.50 & - & - & 16.65 & 27.14 & - & - & - & - & - & - \\
\hline
\end{tabular}


As expected, the chemical spectra of all examined objects contain major elements that are certainly related to clay and ceramic materials. In first is Si (silica) and Al (alumina). Other elements that are probably components of the clay and ceramics are Fe (iron), $\mathrm{K}$ (potassium), $\mathrm{Ca}$ (calcium), $\mathrm{Na}$ (natrium), Mg (magnesia), and Ti (titanium). Relatively high concentrations of Fe in all objects may be explained by the fact that most of the potter's clays from the Primorye region are saturated with iron oxides [22] (p. 37).

Elements that are not characteristic of the material composition of clay or ceramics but are supposedly related to the alloys' compositions are $\mathrm{Pb}$ (lead), $\mathrm{Sn}$ (tin), and $\mathrm{Cu}$ (copper). They are recognized only on objects with carbonized casting cavities and pouring gates (number 13, 15, 20, 23, 25, 31, 33, and 34). Single objects with the carbonization of a working surface (number 18) are free of $\mathrm{Pb}, \mathrm{Sn}$, or $\mathrm{Cu}$ traces. The $\mathrm{Pb}$ contents in the EDS spectra of six objects varied broadly from $1.59 \%$ up to $52.56 \%$ (Tables 3 and 4 ). Pb forms the most significant peaks on the spectrograms. There are separate, distinguished peaks of $\mathrm{Pb}$ (Figure 8a) and joint Pb-Si peaks (Figure 8b). The Pb-Si peaks are registered in most cases.
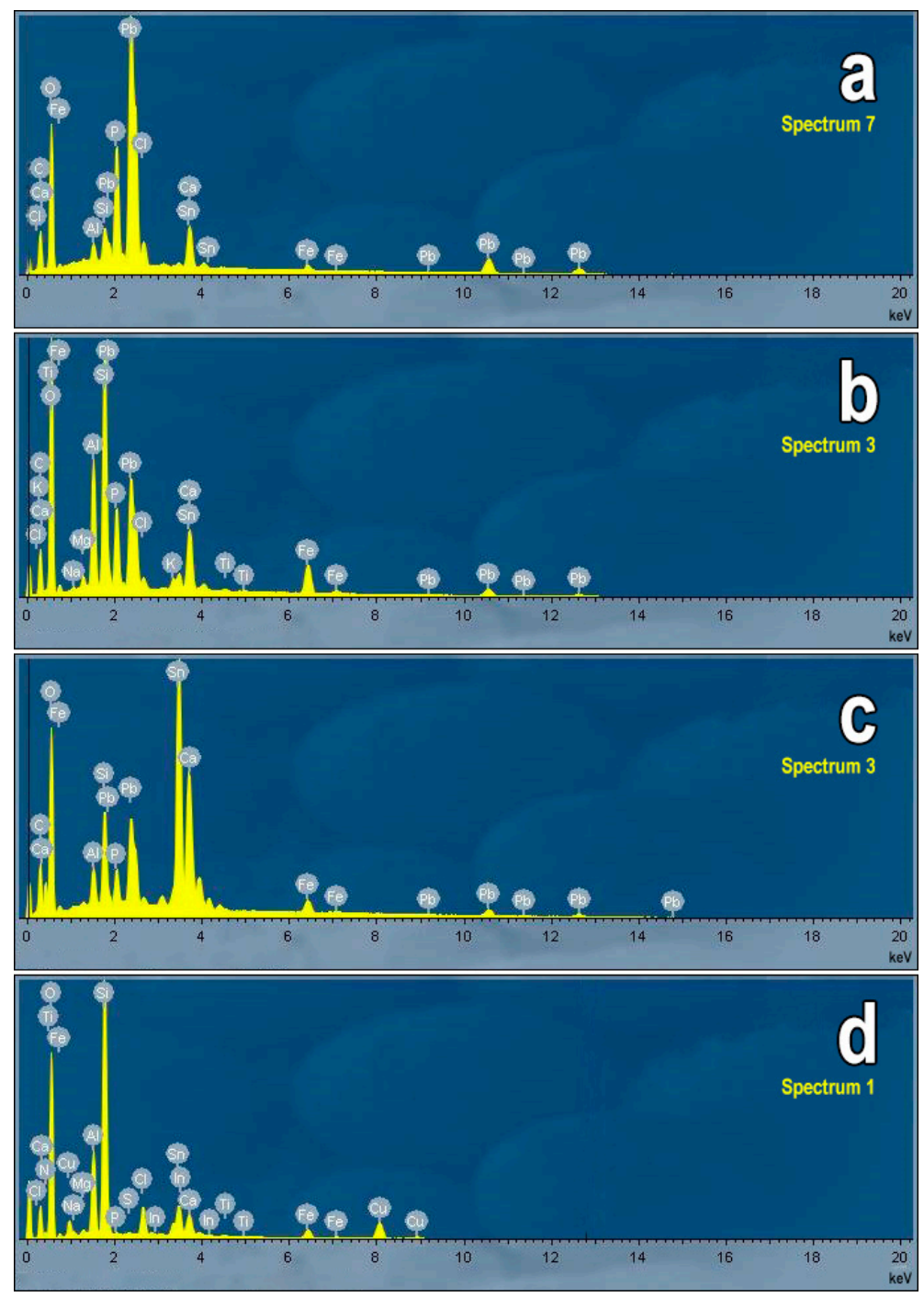

Figure 8. Kruglaya Dolina site. Selected EDS spectra of examined objects. (a) Object number 34, test site 1, spectrum 7. (b) Object number 34, test site 2, spectrum 3. (c) Object number 34, test site 1, spectrum 3. (d) Object number 15, test site 1, spectrum 1.

The Sn contents in the EDS spectra of five objects varied from $3.61 \%$ to $33.86 \%$ (Tables 3 and 4; Figure 8c). The $\mathrm{Sn}$ traces were observed via screening pXRF examination confirmed with the SEM-EDS results (Figure 9). Sn was distributed over the working 
surface of the casting mold as inhomogeneous inclusions up to $400 \mu \mathrm{m}$ in length. The distribution and size of the inclusions suggest their origin as remnants of cast alloys containing Sn.

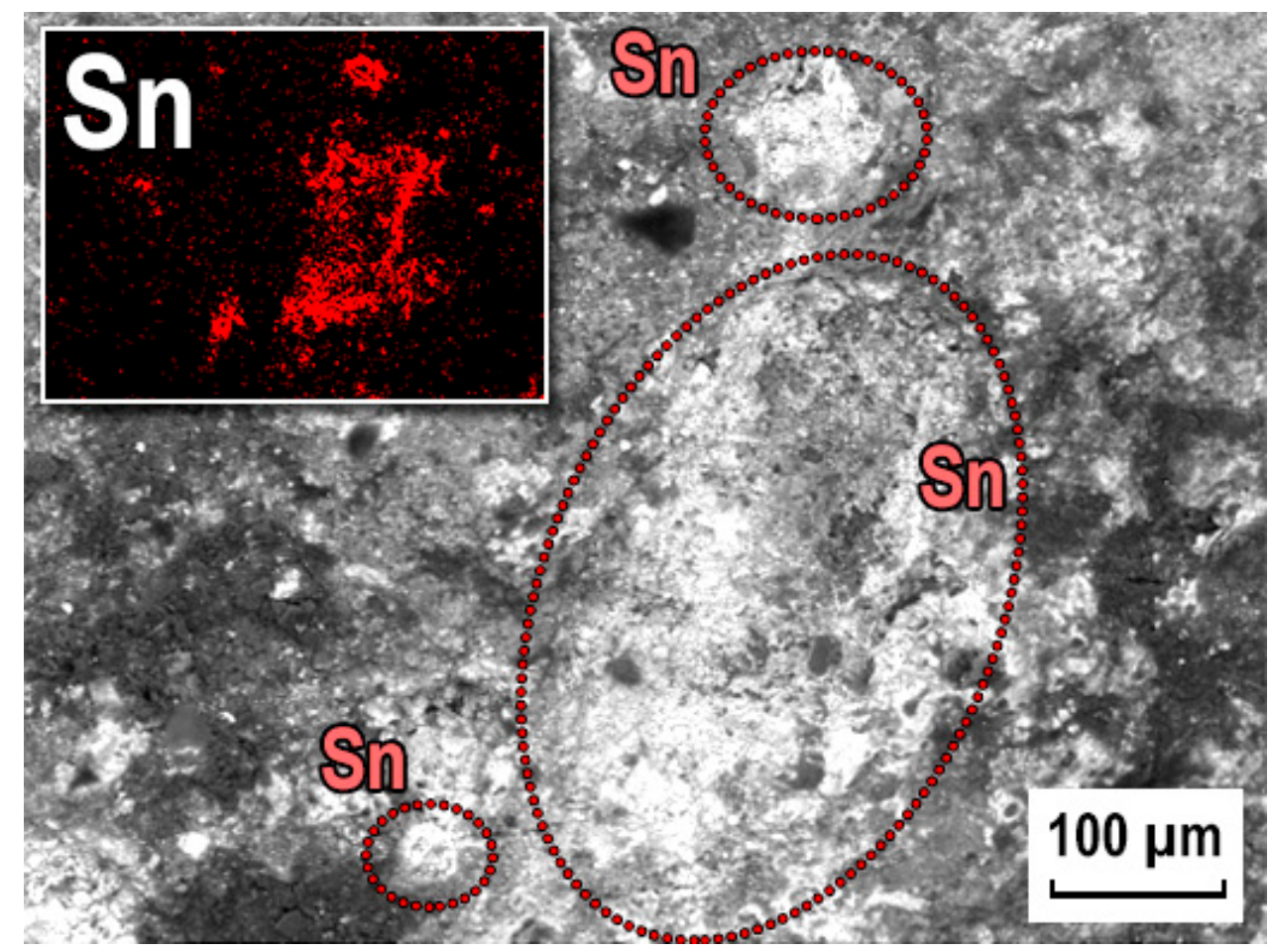

Figure 9. Kruglaya Dolina site. SEM image of the working surface of the mold (object number 34) with $\mathrm{Sn}$ traces on EDS mapping.

The $\mathrm{Cu}$ contents in the EDS spectra of three objects varied from $2.02 \%$ to $10.69 \%$ (Table 3; Figure 8d). It must be noted that $\mathrm{Cu}$ was recognized also for object number 20 in concentrations less than $1.0 \%$ (Table 2).

It may be emphasized that no traces of $\mathrm{Pb}, \mathrm{Sn}$, and $\mathrm{Cu}$ are recognized in the EDS spectra from the non-carbonized working sides of object number 17, 16, 22, and 26 and in the EDS spectra from the "unworking" (back) side of object number 15.

As (arsenic) was detected in five objects, but only in one object (number 13) was the contents of this element $\geq 1.0 \%$ (Table 3). In other objects, the concentration of As was measured as hundredths and tenths of a percent. Arsenic, which is not a characteristic element of clay and ceramics chemical compositions, may be interesting as an impurity in copper ores or as an added component of alloy [11] (pp. 60-61, 74), [23,24]. Taking into account the concentration of As in object number 13, it seems likely that this element is an alloy component.

The EDS method shows the presence of Zn only for object number 20 within the concentration range of $0.13-0.33 \%$ The data on $\mathrm{Zn}$ intensities on the EDS spectra and the results obtained by a handheld pXRF analyzer are slightly different but not contradictory. The pXRF analyzes an extensive surface area so that the average mass concentration of $\mathrm{Zn}$ has a low value but demonstrates the stable presence of this element on the molds' working surface. The surface analysis by the SEM method is more accurate, and analyzing local areas does not allow for determining a stable presence of $\mathrm{Zn}$ in all measurements. Therefore, it is fair to conclude that $\mathrm{Zn}$ is most likely strongly dispersed in low concentrations on the molds' working surfaces as an element of the ceramics.

$\mathrm{P}$ (phosphorous) can be identified in most of objects examined (Table 3). It is important to note the significant differences in P concentrations in the objects. Relatively high concentrations of $\mathrm{P}(3.22-11.63 \%)$ are detected certainly for the Pb-containing objects 
(number 13, 20, 25, 31, 33, and 34). In other objects, P concentrations are low $(\leq 1.5 \%)$ or extremely low $(\leq 1.0 \%)$. The detection of $\mathrm{P}$ in archaeological ceramics may be due to various reasons and, in particular, to this chemical element penetrating from the soil [25-27]. In this connection, some tendency toward overlapping $\mathrm{P}$ and $\mathrm{Ca}$ concentrations may be noted. Pb-containing objects, number 13, 20, 25, 31, and 34, show high concentrations of $\mathrm{P}$ together with enhanced concentrations of $\mathrm{Ca}$ (5.76-19.24\%). In this connection, object number 31 is of special interest. SEM imaging of one of the test areas shows the angular-shaped mineral inclusion of a size of around $40.0 \mu \mathrm{m}$ (Figure 10a). The EDS spectrum of this inclusion is characterized by extremely high concentrations of $\mathrm{P}, 11.34 \%$, and $\mathrm{Ca}, 17.24 \%$ (Figure 10b). Concerning the possible reasons for the mutually high $\mathrm{P}$ and $\mathrm{Ca}$ concentrations, it may be noted that these elements are the main components of some kinds of soil fertilizers. In particular, the orthophosphate $\left(\mathrm{CaPO}_{4}\right)$ used in powder and crystalline forms is one of most widely known and practiced soil additives [28,29]. Taking into account the localization of the archaeological site Kruglaya Dolina in the area of long-termed agricultural activity, it may be thought that high concentrations of $\mathrm{P}$ and $\mathrm{Ca}$, in particular in mineral inclusion form, are due to penetration from the fertilized soil.
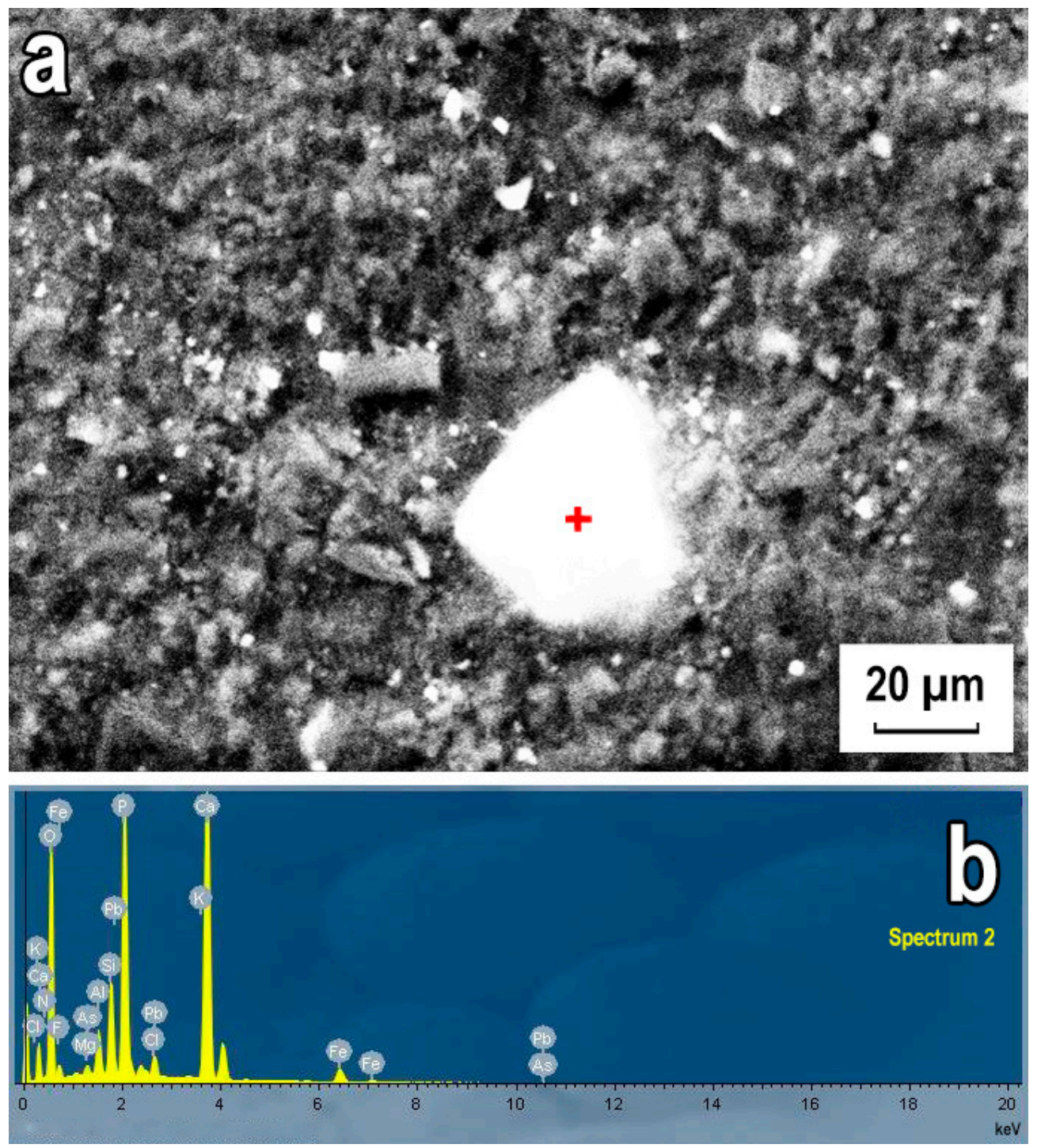

Figure 10. Kruglaya Dolina site. Object number 31. (a) SEM image of a cavity surface microstructure with mineral inclusion containing high concentrations of $\mathrm{P}$ and Ca. "+" marks the EDS spectrum. (b) EDS spectrum of mineral inclusion. 
The constant element recognized in 12 of the 13 examined objects is $\mathrm{Cl}$ (chlorine). Its concentrations are close for different objects (Table 2). It seems likely that the detection of $\mathrm{Cl}$ at archaeological ceramic object surfaces may be explained by its penetration from soil, where this element naturally originates or is the result of human activities [30]. In this connection, it is important to note the relatively high concentrations of $\mathrm{K}$ (potassium) recognized for most examined objects (Table 3). $\mathrm{K}$ is one of the major clays and, consequently, is a material element of ceramics. However, additionally, potassium and chlorine may penetrate the porous ceramics material from the soils. In particular, the source of both elements might be $\mathrm{KCl}$ (potassium chloride), which is well-known as a wide spread soil fertilizer [31]. An explanation for the high $\mathrm{K}$ and $\mathrm{Cl}$ concentrations at the ceramic mold surfaces seems to be the same as that in the case of high $\mathrm{P}$ and Ca concentrations.

The metals $\mathrm{Mn}, \mathrm{Ba}, \mathrm{In}, \mathrm{Zr}$, and Ce appear in EDS spectra of some objects (Tables 3 and 4). According to the data, all of these elements are present in natural clays and soil chemical contents [32-35]. At the same time, In, Mn, Ba are known as the impurities in ores used for bronze-casting [36,37]. Preliminarily, considering the concentrations of elements, $\mathrm{Mn}$ in object number 13, In in object number 15, and Ba in object numbers 20 and 25 may be the ore's impurities.

\section{Discussion and Conclusions}

The use of nondestructive pXRF and SEM-EDS methods for the examination of ancient ceramic casting molds from the Primorye region, southern Russian Far East, resulted in the detection of probable traces of copper alloy. The results obtained by the much more accurate SEM-EDS method verifies the data from the pXRF screening analysis. The metals $\mathrm{Pb}, \mathrm{Sn}$, and $\mathrm{Cu}$ were interpreted as alloy components and were recognized on the surface of ceramics. Importantly, relatively significant concentrations of these elements were found in trace amounts on the molds' working cavity surfaces with evidence of carbonization. Non-carbonized ceramics surfaces were free of traces of $\mathrm{Pb}, \mathrm{Sn}$, and $\mathrm{Cu}$. Our EDS and pXRF data correspond, in general, with the results of other experimental investigations detecting traces of alloy on ceramic molds surfaces [9]. $\mathrm{Pb}, \mathrm{Sn}$, and $\mathrm{Cu}$ certainly show different patterns. For most of the examined objects with carbonized cavity surfaces, significant concentrations of $\mathrm{Pb}$ are detected. The elements $\mathrm{Sn}$ and especially $\mathrm{Cu}$ show more episodic and weak appearances. According to these data, some preliminary notions on probable alloy compositions may be suggested.

Representative series of the objects (numbers 1-5, 8, 13, 20, 25, and 31-34) indicate the usage of alloys ligated with lead (Tables 2 and 3). The instability of silica-tin (Si-Sn) and silica-copper ( $\mathrm{Si}-\mathrm{Cu}$ ) compounds is supposed to be the reason that, in a single case only (object number 13), the co-presence of three elements- $\mathrm{Pb}, \mathrm{Sn}$, and $\mathrm{Cu}-$ are detected. Notably, this case is confirmed by both EDS and pXRF results. Object number 13 is also interesting as the As concentration of $1.5 \%$ allows us to suppose that this element is an alloy component. For object numbers 25, 31, and 34, where the EDS detects Pb and Sn, the presence of $\mathrm{Cu}$ is automatically assumed. These data allow us to assume the usage of multicomponent copper alloys containing $\mathrm{Cu}, \mathrm{Pb}, \mathrm{Sn}$, and $\mathrm{As}$, and $\mathrm{Cu}, \mathrm{Pb}$, and $\mathrm{Sn}$, correspondingly. According to the pXRF results, object numbers 8, 28, and 33 show the co-presence of $\mathrm{Pb}$ from $2.83 \%$ to $6.79 \%$ and $\mathrm{Cu}$ at the tenths of a percent while no $\mathrm{Sn}$ traces were detected. According to EDS, no traces of $\mathrm{Sn}$ and $\mathrm{Cu}$ were recognized in object number 33. Therefore, for the cases of object numbers 8,28 , and 33 , the copper alloy containing $\mathrm{Cu}$ and $\mathrm{Pb}$ is assumed tentatively.

The EDS examination of two objects indicates the supposed usage of alloys not containing lead. These objects show the presence of $\mathrm{Sn}$ and $\mathrm{Cu}$ (number 15), and $\mathrm{Cu}$ only (EDS of number 23). Considering the stability and detection of available Pb-Si compounds, it may be thought that, in these cases, lead initially was not a part of the alloy compositions. Therefore, two kinds of lead-free alloys are assumed - one containing $\mathrm{Cu}$ and $\mathrm{Sn}$, and the other containing $\mathrm{Cu}$. 
As noted in the Introduction, previous investigations on the chemical contents of Bohai period copper alloys based on the examination of bronze artifacts and metal residues from ceramic crucibles has revealed the co-existence of several composition systems. First, there are $\mathrm{Pb}$-containing systems recognized in most of the cases: $\mathrm{Cu}-\mathrm{Pb}, \mathrm{Cu}-\mathrm{Sn}-\mathrm{Pb}, \mathrm{Cu}-\mathrm{Sn}-\mathrm{Pb}-\mathrm{As}$, and $\mathrm{Cu}-\mathrm{Pb}$-As. It was determined that $\mathrm{Pb}$ is used widely for the ligation of copper alloys in the southern Russian Far East since the beginning of the 1st mil AD. Non Pb-containing systems such as $\mathrm{Cu}, \mathrm{Cu}-\mathrm{Sn}$, and $\mathrm{Cu}-\mathrm{As}$ were also distinguished. The multicomponent alloys were practiced most widely during the Bohai period. Importantly, Zn was not used as a ligating component of copper alloys at that time. Zn-containing alloys became widespread over the territory of southern Russian Far East some time later, in XII-XIII c. AD [11] (pp. 60-62, 84-85), [12,13].

As may be seen, the results of the EDS and pXRF examination of ceramic molds from the Kruglaya Dolina site, are consistent, in general, with the referenced data. The supposed variants of the alloy compositions distinguished through the set of main metals $(\mathrm{Cu}-\mathrm{Pb}-\mathrm{Sn}$, $\mathrm{Cu}-\mathrm{Pb}-\mathrm{Sn}-\mathrm{As}, \mathrm{Cu}-\mathrm{Pb}, \mathrm{Cu}$, and $\mathrm{Cu}-\mathrm{Sn}$ ) do not contradict to the composition of Bohai period copper alloys. Note that the absence of convincing traces of $\mathrm{Zn}$ on the casting mold surfaces as a probable alloy component is also consistent with earlier research results. It has to be emphasized that no traces of precious metals, in particular, Ag and $\mathrm{Au}$, were detected on the molds' surfaces. The ceramic molds were used just for the bronze casting.

Some concluding remarks may be stated. First, our investigation confirmed the possibility of using archaeological ceramic casting molds as potential sources of information about ancient copper-alloys contents. Applying the pXRF and SEM-EDS methods allows us to detect the presence of $\mathrm{Pb}, \mathrm{Cu}, \mathrm{Sn}$, and As as probable alloy components on mold working surfaces. An essential condition for recognizing these elements is a carbonized state of the ceramic surface, certainly indicating the casting process. Even though the contents of the alloys may be determined at a relative qualitative level but not at an accurate quantitative one, the data obtained are of interest in the context of ancient bronze-casting. The materials presented were used as a pilot physicochemical examination of ancient ceramic casting molds from southern Russian Far East. Research concerning the unique case of the bronzecasting workshop remains at the Bohai period Kruglaya Dolina site contributes to the widening general database of copper alloys practiced in early medieval times.

Author Contributions: Conceptualization, I.S.Z.; methodology, I.S.Z. and I.Y.B.; investigation, I.S.Z. and I.Y.B.; writing —original draft preparation, I.S.Z.; writing—review and editing, I.Y.B.; visualization, I.Y.B.; project administration, I.S.Z. All authors have read and agreed to the published version of the manuscript.

Funding: The research was financially supported by the Russian Science Foundation (project No. 2018-00081).

Institutional Review Board Statement: Not applicable.

Informed Consent Statement: Not applicable.

Data Availability Statement: Not applicable.

Acknowledgments: The SEM-EDS examination of sampled objects was executed in the Electron Microscopy Centre of National Scientific Marine Biology Centre, Far Eastern Branch of Russian Academy of Sciences. The authors thank the Head of Electron Microscopy Centre Denis V. Fomin for the technical assistance and support. The authors are grateful to Professor of Archaeology Peter D. Jordan (Lund University, Lund, Sweden) and Richard L. Bland (University of Oregon, Eugene, OR, USA) for their assistance with the English corrections and for their helpful edits and comments.

Conflicts of Interest: The authors declare no conflict of interest.

\section{References}

1. Lopatina, O.A. Technological Characteristic of Casting Moulds from D'yakovo Hillfort. Russ. Archaeol. 2002, 4, 111-112. (In Russian)

2. Krenke, N.A.; Tavlintseva, E.Y. Casting Moulds from the D'yakovo Hillfort. Russ. Archaeol. 2002, 4, 90-110. (In Russian) 
3. Stoltman, J.B.; Jing, Z.; Tang, J.; Rapp, G. Ceramic Production in Shang Societies of Anyang. Asian Perspect. 2009, 48, 182-203. [CrossRef]

4. Liu, S.; Wang, K.; Cai, Q.; Chen, J. Microscopic study of Chinese bronze casting moulds from the Eastern Zhou period. J. Archaeol. Sci. 2013, 40, 2402-2414. [CrossRef]

5. Durakov, I.A.; Kobeleva, L.S. Technical Ceramics of Krotovo Culture (Central Baraba). Bull. Tomsk. State Univ. Hist. 2017, 49, 23-25. (In Russian) [CrossRef]

6. Molodin, V.I.; Mylnikova, L.N.; Shtertser, N.V.; Durakov, I.A.; Drebushchak, V.A. Thermogravimetry in the Studies of Ancient Technical Ceramics. Chem. Sustain. Dev. 2019, 27, 101-108. [CrossRef]

7. Zong, Y.; Yao, S.; Lang, J.; Chen, X.; Fan, J.; Sun, Z.; Duan, X.; Li, N.; Fang, H.; Zhou, G.; et al. Structural and compositional analysis of a casting mold sherd from ancient China. PLoS ONE 2017, 12, e0174057. [CrossRef] [PubMed]

8. Yan, B.; Liu, S.; Chastain, M.L.; Yang, S.; Chen, J. A new FTIR method for estimating the firing temperature of ceramic bronzecasting moulds from early China. Sci. Rep. 2021, 11, 3316. [CrossRef] [PubMed]

9. Kearns, T.; Martinon-Torres, M.; Rehren, T. Metal to mould: Alloy identification in experimental casting moulds using XRF. Hist. Metall. 2010, 44, 48-58.

10. Garbacz-Klempka, A.; Kwak, Z.; Żak, P.L.; Szucki, M.; Ścibior, D.; Stolarczyk, T.; Nowak, K. Reconstruction of the casting technology in the Bronze Age on the basis of investigations and visualization of casting moulds. Arch. Foundry Eng. 2017, 17, 184-190. [CrossRef]

11. Konkova, L.V. The Bronze-Casting Craft in the Southern Far East of the USSR. The Boundary of the II-I Millennium BC.-XIII Century $A D$; Nauka Press: Leningrad, Russia, 1989. (In Russian)

12. Gelman, E.I.; Kojima, Y. The Bohai bronze foundry in the Ilistaya River valley. Bull. Tomsk. State Univ. Hist. 2013, 2, 26-30. (In Russian)

13. Gelman, E.I.; Astashenkova, E.V.; Buravlev, I.Y. Bronze-casting production in the Bohai (by data from archaeological research on Kraskinskoe walled town site). Multidiscip. Stud. Archaeol. 2017, 3, 141-179. (In Russian)

14. Thuesen, I.; Oldenburg, E.; Iørgensen, K. Microscope Section Analysis of Pottery. Paléorient 1989, 15, 273-278. [CrossRef]

15. Santacreu, D.A. Identifying spathic calcite recipe in archaeological ceramics: Possibilities and limitations. Ceramica 2014, 60, 379-391. [CrossRef]

16. Tite, M.S.; Freestone, I.C. The use of scanning electron microscopy in the technological examination of ancient ceramics. In Archaeological Ceramics; Smithsonian Institution Press: Washington, DC, USA, 1982; pp. 109-120.

17. Tite, M.S. The Impact of Electron Microscopy on Ceramic Studies. Proc. Br. Acad. 1992, 22, 111-131.

18. Shepard, A.O. Ceramic for the Archaeologist; Carnegie Institute: Washington, DC, USA, 1985.

19. Rice, P.M. Pottery Analysis: A Sourcebook; Chicago University: Chicago, IL, USA, 1987.

20. Zhushchikhovskaya, I.S. Experience of Using Scanning Electron Microscopy in the Study of Archaeological Ceramics from the Southern Far East. Trans. Inst. Hist. Mater. Cult. 2017, 16, 75-91. (In Russian)

21. Sahlén, D. Ceramic Evidence from Non-ferrous Metallurgy in the Mälgren Valley during the Viking Age. In Prehistoric Pottery across the Baltic; BAR International Series 2785; Archaeopress: Oxford, UK, 2016; pp. 75-80.

22. Zhushchikhovskaya, I.S. Prehistoric Pottery-Making of the Russian Far East; BAR International Series 1434; Archaeopress: Oxford, UK, 2005.

23. Lechtman, H. Arsenic Bronze: Dirty Copper or Chosen Alloy? A View from the Americas. J. Field Archaeol. 1996, 4, 477-514. [CrossRef]

24. Radivodjevic, M.; Roberts, B.W.; Pernicka, E.; Stos-Gale, Z.; Martinón-Torres, M.; Rehren Th Bray, P.; Brandherm, D.; Ling, J.; Mei, J.; Vandkilde, H.; et al. The Provenance, Use and Circulation of Metals in the European Bronze Age: The State of Debate. J. Archaeol. Res. 2019, 27, 131-185. [CrossRef]

25. Duma, G. Phosphate Content of Ancient Pots as Indication of Use. Curr. Anthropol. 1972, 13, 127-130. [CrossRef]

26. Freestone, I.C.; Middleton, A.P.; Meeks, N.D. Significance of phosphate in ceramic bodies: Discussion of paper by Bollong et al. Archaeol. Sci. 1994, 21, 425-426. [CrossRef]

27. Maritan, L.; Angelini, G.; Mazzoli, C.; Saracino, M. Secondary phosphates in the ceramic materials from Frattesini (Rovigo, North-Eastern Italy). J. Cult. Herit. 2009, 10, 144-151. [CrossRef]

28. Hagin, J. Availability of Dicalcium Phosphate to Plants When Applied in Various Forms. Plant Soil. 1958, 10, 101-113. [CrossRef]

29. McBeath, T.M.; Lombi, E.; McLaughlin, M.J.; Bunemann, E.K. Pyrophosphate and orthophosphate addition to soils: Sorption, cation concentrations, and concentrations, and dissolved organic carbon. Aust. J. Soil Res. 2007, 45, 237-245. [CrossRef]

30. White, P. Chloride in soils and its uptake and movement within the plant: A review. Ann. Bot. 2001, 88, 967-988. [CrossRef]

31. Pereira, D.G.C.; Santana, I.A.; Megda, M.M.; Megda, M.X.V. Potassium chloride: Impacts on soil microbial activity and nitrogen mineralization. Ciênc. Rural. 2019, 49, e20180556. [CrossRef]

32. Kuleff, I.; Djingova, R. Provenance study of pottery; choice of elements to be determined. Rev. d'Archéom. 1996, 20, 57-67. [CrossRef]

33. Eskenazy, G.M. On the geochemistry of indium in coal-forming process. Geochim. Cosmochem. Acta 1980, 44, 1023-1027. [CrossRef]

34. Santos, J.O.; Munita, C.S.; Valerio, M.E.G.; Vergne, C.; Oliveira, P.M.S. Determination of trace elements in archaeological ceramics and application of Kernel Density Estimates: Implications for the definition of production locations. J. Radioanal. Nucl. Chem. 2006, 269, 441-445. [CrossRef] 
35. Liu, Y.-H.; Shaheen, S.M.; Rinklebe, J.; Hseu, Z.-Y. Pedogeochemical distribution of gallium, indium and thallium, their potencial availability and associated risk in highly weathered soil profiles of Taiwan. Environ. Res. 2021, 197, 110-994. [CrossRef] [PubMed]

36. Caley, E.R.; Easby, D.T. Indium as an impurity in ancient Western Mexican tin and bronze artifacts and in local tin ore. Science 1967, 155, 686-687. [CrossRef]

37. Grigoriev, S. Metallurgical Production in Northern Eurasia in the Bronze Age; Archaeopress Publishing Ltd.: Oxford, UK, 2015. 